\title{
Shallow two-component gravity-driven flows with vertical variation
}

\author{
Julia Kowalski ${ }^{2}$ and Jim N. McElwaine ${ }^{1,2,3, \dagger}$ \\ ${ }^{1}$ Department of Applied Mathematics and Theoretical Physics, University of Cambridge, \\ Wiberforce Road, Cambridge CB3 OWA, UK \\ ${ }^{2}$ WSL Institute for Snow and Avalanche Research SLF, Flüelastrasse 11, \\ CH-7260 Davos Dorf, Switzerland \\ ${ }^{3}$ Planetary Science Institute, Tucson, AZ 85719, USA
}

(Received 5 April 2011; revised 27 July 2012; accepted 3 October 2012)

Gravity-driven geophysical mass flows often consist of a heterogeneous fluid-solid mixture. The complex interplay between the components leads to phenomena such as lateral levee formation in avalanches, or a granular front and an excess fluid pore pressure in debris flows. These effects are very important for predicting runout and the forces on structures, yet they are only partially represented in simplified shallow flow theories, since rearrangement of the mixture composition perpendicular to the main flow direction is neglected. In realistic flows, however, rheological properties and effective basal drag may depend strongly on the relative concentration of the components. We address this problem and present a depth-averaged model for shallow mixtures that explicitly allows for rearrangement in this direction. In particular we consider a fluid-solid mixture that experiences bulk horizontal motion, as well as internal sedimentation and resuspension of the particles, and therefore resembles the case of a debris flow. Starting from general mixture theory we derive bulk balance laws and an evolution equation for the particle concentration. Depth-integration yields a shallow mixture flow model in terms of bulk mass, depth-averaged particle concentration, the particle vertical centre of mass and the depth-averaged velocity. This new equation in this model for the particle vertical centre of mass is derived by taking the first moment, with respect to the vertical coordinate, of the particle mass conservation equation. Our approach does not make the Boussinesq approximation and results in additional terms coupling the momentum flux to the vertical centre of mass. The system is hyperbolic and reduces to the shallow-water equations in the homogeneous limit of a pure fluid or perfect mixing. We highlight the effects of sedimentation on resuspension and finally present a simple friction feedback which qualitatively resembles a large-scale experimental debris flow data set acquired at the Illgraben, Switzerland.

Key words: geophysical and geological flows, multiphase and particle-laden flows, particle/fluid flows, shallow water flows 
Flow direction
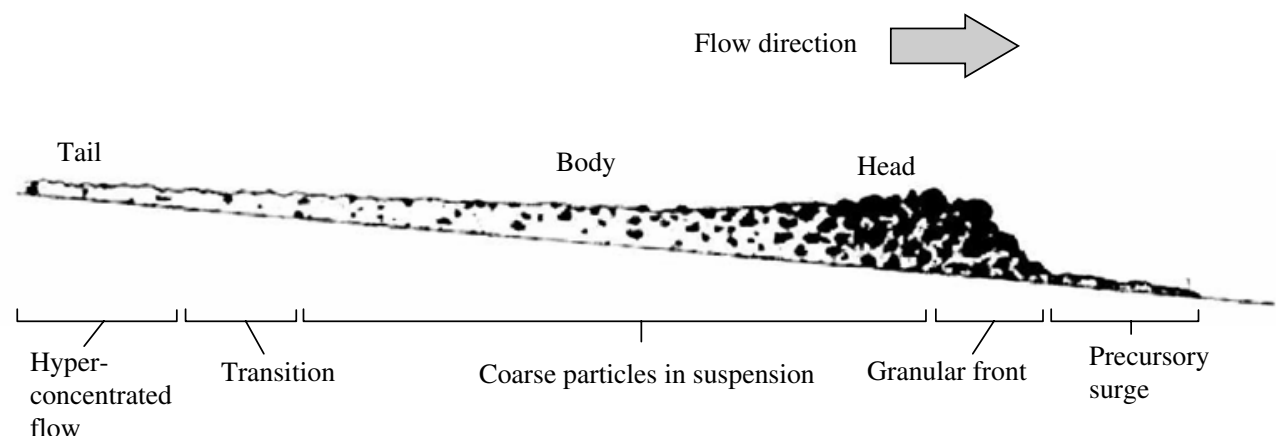

FIGURE 1. Schematic of a granular-front debris flow. A precursory water surge is followed by the head consisting of a mixture of settled big particles and fluid. After the passage of the front the average size of the particles gets smaller and the number of suspended particles increases.

\section{Introduction}

Geophysical mass flows such as avalanches, rockfalls and debris flows have enormous destructive power. They are a major hazard in mountainous regions all over the world. Although these flows have many differences they all give rise to equations with a similar structure, that is mass conservation for the different components and momentum conservation that is a balance between inertia, gravity and drag. If the surface over which the flow occurs is sufficiently smooth then gravity also acts to smooth out the flow and eventually the shape of the flowing body exhibits a shallow flow geometry, meaning that properties vary much more slowly parallel to the slope than perpendicularly. This enable an expansion which can be framed as a depthaveraged model. The subtleties of this are sometimes overlooked. For example a direct expansion to second order gives Benny's equation (Benny 1973) which is unstable. To accurately predict the onset of instabilities such as roll waves a complicated analysis is necessary (Scheid, Ruyer-Quil \& Manneville 2006). Despite these difficulties shallow geophysical flow models have been derived for and applied to many different mass flow phenomena such as avalanches, sub-aquatic and sub-aerial debris flows, rockfalls, and pyroclastic flows to name a few (see for example Savage \& Hutter 1989; Iverson \& Denlinger 2001; Gray 2002; Pitman \& Le 2005; Iverson 2009; Christen, Kowalski \& Bartelt 2010).

A characteristic of geophysical flows is that they are highly heterogeneous and consist of many different fluid, solid and gaseous constituents which experience strong coupling forces. Mathematical geophysical flow theories are therefore conceptually idealized either as a homogeneous continuum with an apparent non-Newtonian rheology characterizing the particular type of flow involved, or as a multi-component mixture that explicitly accounts for the complex coupling. Models of the first type can work well in situations where their assumptions about the vertical distributions of components are valid. However, whenever the internal rearrangement of the components is important, such as in initiation and stopping processes, they will have difficulties accurately describing the flow. Since these models do not include this rearrangement they also cannot model such phenomena as the formation of a granular front or changes in the excess fluid pore pressure due to suspended particles (see figure 1). Models that capture the multi-component nature of the flow allow for a change in the mixture composition. Either the mixture character is considered when 
determining the bulk stress composition (see Iverson \& Denlinger 2001; Denlinger \& Iverson 2001; Iverson 2009; Berzi \& Jenkins 2009) or relative, horizontal motion is explicitly accounted for (see Pitman \& Le 2005). Vertical rearrangement within the mixture, however, is often neglected as it cancels out during common depth-integration procedures, despite its great importance even in shallow flows. In debris flows for instance, sedimentation, segregation and resuspension processes lead to a redistribution of the mixture components that on the one hand feeds back on the bulk dynamics and on the other hand plays a crucial role in the initiation and deposition regime.

We address this problem and propose an extended, depth-averaged, shallow mixture flow model, that explicitly accounts for a vertical rearrangement of its components. In particular we will consider a fluid-solid mixture that is subject to bulk horizontal motion as well as internal sedimentation and resuspension processes. In the geophysical context these flows can be thought of mobilized sediments and rocks saturated by interstitial water and hence resemble sediment transport or debris flows. However, our derivation and arguments are very general and can directly be transferred to other shallow multi-component mixtures, such as powder snow avalanches, pyroclastic flows or polydisperse dry granular flows. Our proposed model has similar objectives to Iverson's theory (Iverson 1997, 2009), that is to include the effects of changing pore pressure on flow dynamics. It can be seen as an extension of the classical theory of Savage \& Hutter (1989) and as an approximation to the two-component theory of Pitman \& Le (2005). If we were to consider the full Mohr-Coulomb closure the proposed model would indeed reduce to the Savage-Hutter equations in the limit of two similar constituents. On the other hand, the proposed model is less comprehensive than the Pitman \& Le approach as we project the systems dynamics onto the bulk velocity rather than separate velocities for each phase. This, however, results in the clear benefit of arriving at a well-posed and hyperbolic system of equations without the inconvenient elliptic degeneracy that can be observed in the Pitman \& Le theory. Physically this occurs because the two phases are very strongly coupled and the system can be well approximated by a bulk velocity and an algebraic closure for the velocity difference; this is the essence of our approach. Instead of taking a very process-orientated viewpoint, which led to the formulation of very specialized extra equations in Iverson's model, we try to put focus on a methodology to capture the simplest rearrangement within a shallow mixture. In that sense our contribution is to be seen as a complementary extension to existing theories that will allow great accuracy with regard to field observations.

Though we wish to allow for a varying mixture density, we still want to make use of the simplifying framework of depth-averaging, which results in a model of reduced dimensionality. We achieve this by starting from general mixture theory and derive bulk balance laws, as well as an evolution equation for the vertical particle profile. Similarly to many other geophysical mass flow models, we then employ the shallowwater approximation and depth average our system. In the case of non-constant mixture density, the depth-averaged bulk momentum balance explicitly depends on the vertical mass centre. A closure is derived along the lines of a moment-based approach that projects the particle profile onto the vertical centre of mass. This procedure allows a separate consideration of horizontal (parallel to the slope) bulk flow dynamics and vertical (normal to the slope) internal processes. Both are coupled through the bulk pressure and gravitational and frictional source terms.

To highlight the innovation in our model we discuss it in the context of the very simplest approximations. The model presented in this paper is therefore considerably simpler than the state of the art. We do discuss in the appropriate sections, however, 
how more complicated closures and possible generalizations can be incorporated. Although most of the arguments are formed for the specific case of fluid-solid mixtures, our approach, namely tracking the vertical distribution of the mixture within a shallow flow framework, can be widely applied to many geophysical flows including turbidity currents and powder snow avalanches. The approach can also be applied to flows such as rockfalls where there is no fluid component, but instead the different components are different classes of rock sizes.

In $\S 2$, we discuss the general framework of mixture theory for a multi-component fluid and specify this for the case of a binary liquid-solid mixture. We derive an evolution equation for the particle volume fraction that will later serve as the basis for an evolution equation of the vertical centre of mass. Section 3 is devoted to the derivation of the depth-averaged system. The basic concept of depth-averaging in the classical constant-density case is generalized to a non-constant mixture density by considering higher moments of the basic balance laws. In $\S 4$ we discuss the mathematical properties of our theory and the physical relevance of the extended shallow geophysical flow equations. We conclude with a final discussion.

\section{Governing equations}

\subsection{General mixture theory}

Let us consider a multi-component mixture denoted by $i \in\{1, \ldots, N\}$. The individual mass and momentum equations are given by

$$
\begin{aligned}
\partial_{t} \rho_{i}+\nabla \cdot\left(\boldsymbol{u}_{i} \rho_{i}\right) & =0, \\
\partial_{t}\left(\boldsymbol{u}_{i} \rho_{i}\right)+\nabla\left(\boldsymbol{u}_{i} \boldsymbol{u}_{i} \rho_{i}\right) & =-\nabla \sigma_{i}+\rho_{i} \boldsymbol{g}+\boldsymbol{I}_{i} .
\end{aligned}
$$

For details on the derivation of these equations see for instance Ishii \& Hibiki (2006) and Drew \& Passman (1998). The (mass) density of component $i$ is denoted by $\rho_{i}$ and its velocity $\boldsymbol{u}_{i}$. The total stress acting on component $i$ is given by the symmetric tensor $\sigma_{i}$, and $\boldsymbol{I}_{i}$ is the total force acting on this component from the other components; $\boldsymbol{g}$ is the acceleration due to gravity. Momentum conservation implies $\sum_{i} \boldsymbol{I}_{i}=\mathbf{0}$. There is no net mass transfer between the constituents and hence no source term in the mass conservation equation (2.1a). As we consider gravity-induced geophysical mass flows, the general reference frame will be given by an inclined coordinate system with slope angle $\xi$. Hence, both components of the gravitational acceleration $\boldsymbol{g}$ are nontrivial in general. The $x$-coordinate measures downslope and the $y$-coordinate measures vertically, by which we mean normal to the slope. Starting from this theoretical framework we will now derive equations that capture sedimentation and resuspension of particles in a fluid. The general framework of this derivation has previously been proposed and used to derive the structurally similar segregation-remixing model for bi-dispersed granular matter with and without background fluid (Gray \& Thornton 2005; Gray \& Chugunov 2006; Thornton, Gray \& Hogg 2006).

Balance laws for the bulk of the flow are derived by summing up the individual mass and momentum equations. This results in

$$
\begin{aligned}
\partial_{t} \rho+\nabla \cdot(\boldsymbol{u} \rho) & =0, \\
\partial_{t}(\boldsymbol{u} \rho)+\nabla(\boldsymbol{u} \boldsymbol{u} \rho) & =-\nabla \sigma+\rho \boldsymbol{g},
\end{aligned}
$$

in which $\sigma=\sum \sigma_{i}$ is the bulk stress and $\rho=\sum \rho_{i}$ the bulk density. The barycentric (mass-averaged) velocity $\boldsymbol{u}$ is defined as $\boldsymbol{u} \equiv \sum\left(\rho_{i} / \rho\right) \boldsymbol{u}_{i}$. For the equation of state we 
write

$$
\sum_{i} \frac{\rho_{i}}{\varrho_{i}}=v .
$$

Here, $\varrho_{i}$ denotes the constant intrinsic material density in unit mass per volume of component $i$ and $v$ is a function of space, which denotes the percentage of space filled by all the considered components. For a saturated mixture of particles in a fluid we would have $v=1$ inside the flow, and $v=0$ outside the flow. When considering the granular components only, $v$ stands for the granular volume fraction. In both cases we assume that in a material frame convected with the bulk velocity $\boldsymbol{u}, \boldsymbol{v}$ is constant. Differentiating (2.3) with respect to time $t$ and substituting in mass conservation equation $(2.1 a)$ yields $\boldsymbol{\nabla} \cdot\left(\sum_{i}\left(\rho_{i} / \varrho_{i}\right) \boldsymbol{u}_{i}\right)=0$, where $\left(\rho_{i} / \varrho_{i}\right)$ is the apparent volume fraction of component $i$. Henceforth we will assume that the flow is fully saturated within a well-defined region between the slope and the free surface, that is $v \equiv 1$. This implies that within the flowing body the volume fractions $\rho_{i} / \varrho_{i}$ and mass concentrations $\rho_{i} / \rho$ of the components respectively sum up to one:

$$
\sum_{i} \frac{\rho_{i}}{\varrho_{i}}=1, \quad \sum_{i} \frac{\rho_{i}}{\rho}=1 .
$$

Owing to different intrinsic densities $\varrho_{i}$ of the components, the volume fractions will generally be different from the corresponding mass fractions $\rho_{i} / \rho \neq \rho_{i} / \varrho_{i}$. Hence, we have to distinguish between the volume-averaged velocity field $\boldsymbol{v} \equiv \sum_{i}\left(\rho_{i} / \varrho_{i}\right) \boldsymbol{u}_{i}$ which is divergence free $(\boldsymbol{\nabla} \cdot \boldsymbol{v}=0)$, and its barycentric counterpart $\boldsymbol{u} \equiv \sum\left(\rho_{i} / \rho\right) \boldsymbol{u}_{i}$ with a non-trivial divergence. In the subsequent analysis we formulate the bulk mixture model in terms of the barycentric velocity $\boldsymbol{u}$.

\subsection{A binary fluid-solid mixture model}

The previous considerations have been general. Henceforth we restrict our analysis to a binary mixture of solid particles $(i=p)$ and a fluid component $(i=f)$. We define $\alpha$, the volume fraction of the solids $\alpha \equiv \rho_{p} / \varrho_{p}$, hence apparent density and constant intrinsic density are related by

$$
\rho_{p}=\alpha \varrho_{p}, \quad \rho_{f}=(1-\alpha) \varrho_{f} .
$$

In order to account for sedimentation and resuspension processes within the flowing body, we want to explicitly allow for a small relative motion between the solids and the surrounding fluid. Let us consider the solids mass conservation written in terms of the barycentric velocity $\boldsymbol{u}$ and the slip velocity $\boldsymbol{d} \equiv \boldsymbol{u}_{p}-\boldsymbol{u}_{f}$. With

$$
\boldsymbol{u}_{p}=\boldsymbol{u}+\frac{\rho_{f}}{\rho} \boldsymbol{d} \quad \text { and } \quad \boldsymbol{u}_{f}=\boldsymbol{u}-\frac{\rho_{p}}{\rho} \boldsymbol{d},
$$

the individual mass balances are

$$
\begin{gathered}
\partial_{t} \rho_{p}+\nabla \cdot\left(\boldsymbol{u} \rho_{p}\right)=-\nabla \cdot\left(\frac{\rho_{p} \rho_{f}}{\rho} \boldsymbol{d}\right), \\
\partial_{t} \rho_{f}+\nabla \cdot\left(\boldsymbol{u} \rho_{f}\right)=\nabla \cdot\left(\frac{\rho_{p} \rho_{f}}{\rho} \boldsymbol{d}\right) .
\end{gathered}
$$

Following several authors (Pitman \& Le 2005; Iverson 2009; Pailha \& Pouliquen 2008), we neglect a viscous contribution to the fluid stress tensor and consider pressure only: $\nabla \sigma_{f}=\nabla p_{f}$. Furthermore we split the isotropic pressure $p_{f}$ into a purely 
hydrostatic contribution $p_{h}$ and its deviation $p_{e}$, which is commonly also called the excess pore pressure. Hence, $\nabla \sigma_{f}=\nabla p_{f}=\nabla\left(p_{h}+p_{e}\right)$. We are assuming that the slip velocity $\boldsymbol{d}$ is small so the momentum coupling can be formulated in terms of a buoyancy force plus a drag force, hence $\boldsymbol{I}_{f}=-\boldsymbol{I}_{p}=\left(\rho_{p} \rho_{f} / \rho\right)(\boldsymbol{d} / \tau)+\alpha \nabla p_{h}$, where $\tau$ is a viscous relaxation time scale. The individual components momentum equations transform into

$$
\begin{aligned}
& \rho_{p}\left(\partial_{t} \boldsymbol{u}_{p}+\boldsymbol{u}_{p} \nabla \boldsymbol{u}_{p}\right)=-\nabla \sigma_{p}+\rho_{p} \boldsymbol{g}-\frac{\rho_{p} \rho_{f}}{\rho} \frac{\boldsymbol{d}}{\tau}-\alpha \nabla p_{h}, \\
& \rho_{f}\left(\partial_{t} \boldsymbol{u}_{f}+\boldsymbol{u}_{f} \nabla \boldsymbol{u}_{f}\right)=-\nabla\left(p_{e}+p_{h}\right)+\rho_{f} \boldsymbol{g}+\frac{\rho_{p} \rho_{f}}{\rho} \frac{\boldsymbol{d}}{\tau}+\alpha \nabla p_{h} .
\end{aligned}
$$

On the left-hand side we again have the convective terms. The right-hand side of the particle momentum equation is composed of the particle stress, the gravitational acceleration and the two contributions to the coupling terms, namely buoyancy and viscous drag. The right-hand side of the fluid equation has the same general structure; however in this the fluid stress hydrostatic and excess pore pressure contributions are separated out.

\subsection{Evolution of the particle volume fraction}

Subtracting the individual momentum balance equations $(2.8 a, b)$ from each other and neglecting quadratic terms in $\boldsymbol{d}$ yields an evolution equation for the slip velocity $\boldsymbol{d}$

$$
\partial_{t} \boldsymbol{d}+\boldsymbol{d} \cdot \nabla \boldsymbol{u}+\boldsymbol{u} \cdot \nabla \boldsymbol{d}=-\frac{1}{\rho_{p}} \nabla p_{p}+\frac{1}{\rho_{f}} \nabla p_{e}+\frac{1-\delta}{\varrho_{f}} \nabla p_{h}-\frac{\boldsymbol{d}}{\tau},
$$

where $\delta \equiv \varrho_{f} / \varrho_{p}$ is the intrinsic density ratio. We consider cases in which a characteristic slip velocity due to relative motion of the components is small in comparison to the horizontal bulk flow speed $\left(d_{x} \ll u_{x}\right)$. This is for instance true for mudflows or debris flows in moderately inclined terrain. In Iverson (1997) a scaling argument for the relative discharge is discussed in detail. For negligible topography curvature however, vertical motion is solely due to redistribution of the components. In particular it is not superposed by a bulk motion. Hence we will consider slip of the component in the vertical $y$-direction and neglect horizontal slip $d_{x}$. Convective acceleration of the slip velocity plays a negligible role such that to a first-order approximation we get

$$
d_{y}=\tau\left(-\frac{1}{\rho_{p}} \partial_{y} p_{p}+\frac{1}{\rho_{f}} \partial_{y} p_{e}\right)+g_{y} \tau(1-\delta) .
$$

This approximation is valid when the time scale $\tau$ is much smaller than the times over which the driving terms vary. Here, we substituted for the known hydrostatic pressure gradient $\nabla p_{h}=\varrho_{f} g$. The last term denotes sedimentation due to buoyancy forces. Since the vertical coordinate axis opposes the $y$-component of the gravitational acceleration, $g_{y}$ will be negative. This implies that $g_{y} \tau(1-\delta)$ will be negative for particles that have a higher density than the surrounding fluid $(\delta<1)$. If the intrinsic densities difference is zero $(\delta=0)$ we deal with a neutrally buoyant situation and this term is zero. The first and the second term in (2.10) denote particle pressure and excess fluid pore pressure respectively. If all the particles are in suspension we will have a maximal excess pore pressure and the particle pressure is minimal. When the particles settle down and form a load-bearing matrix $p_{e}$ vanishes and the particles are supported by $p_{p}$. During the settling of particles there is a tendency for the excess pore 
pressure to be transferred to particle pressure, whereas during resuspension the particle pressure is transferred to excess fluid pore pressure. In the following we separate out the constant hydrostatic pressure $p_{h}$ and define excess pore pressure and particle pressure as fractions $X$ and $1-X$ of the reduced hydrostatic pressure $\bar{p}=p-p_{h}$, hence

$$
p_{e}=X \bar{p} \quad \text { and } \quad p_{p}=(1-X) \bar{p} \text {. }
$$

If $X=1$ the complete extra stress is carried by the fluid. This for instance is the case when all particles are suspended. For $X=0$ the complete stress is taken by a load-bearing matrix of particles and the fluid stress $p_{f}$ reduces to the hydrostatic pressure $p_{h}$. We substitute in $\nabla \bar{p}=\left(\rho-\varrho_{f}\right) g_{y}$, and get

$$
d_{y}=\frac{\rho}{\rho_{f} \rho_{p}}\left(\tau X \rho_{p}(1-\delta) g_{y}+\tau \bar{p} \partial_{y} X\right) .
$$

The coefficient $g_{y}(1-\delta)$ accounts for the reduced buoyancy. We define the sedimentation velocity $V \equiv-\tau g_{y}(1-\delta)$ and diffusion parameter $D=\tau \bar{p} /\left(\phi \varrho_{p}\right)$. Here, $\phi$ is the material-dependent maximum packing fraction of the particles. We then substitute (2.12) into the particle mass conservation equation (2.7a), which yields

$$
\partial_{t} \rho_{p}+\nabla \cdot\left(\boldsymbol{u} \rho_{p}\right)=\partial_{y}\left(X V \rho_{p}-D \phi \varrho_{p} \partial_{y} X\right) \text {. }
$$

For zero excess pore pressure, $X=0$, the sedimentation flux vanishes as the complete stress is taken by the solid matrix, whereas $X=1$ yields the sedimentation flux $V \rho_{p}$. A constant stress fraction $X$, however, is unrealistic. Rather, $X$ should depend on the solids volume fraction. In a dilute suspension less stress will be carried by the solids than in a dense packing. We therefore expect a decrease of $X$ with an increasing particle volume fraction and model $X$ according to $X=1-\alpha / \phi$. In this expression $\phi$ again stands for the maximum packing fraction so that we have $X=0$ when the particles have attained a random close packing where they fully bear their own weight (minus buoyancy). For monodisperse glass beads $\phi$ is between $(0.59$ and 0.74$)$ depending on the optimality of the packing. For polydisperse natural mixtures it may be higher. Substituting this closure for $X$ into (2.13) and dividing it by the particle intrinsic density $\varrho_{p}$ yields the following evolution equation for the solids volume fraction $\alpha$ :

$$
\partial_{t} \alpha+\nabla \cdot(\boldsymbol{u} \alpha)=\partial_{y}\left[V \alpha\left(1-\frac{\alpha}{\phi}\right)+D \partial_{y} \alpha\right] .
$$

The advective flux term on the left-hand side corresponds to transport with the barycentric velocity field $\boldsymbol{u}$. The first term on the right-hand side represents nonlinear sedimentation of Richardson-Zaki type (Richardson \& Zaki 1954), and the second term represents diffusion of the particles, including resuspension. If the particles and the surrounding fluid have the same intrinsic density, the density ratio will be one $(\delta=1)$ and the sedimentation velocity vanishes. Related concepts to analyse nonlinear sedimentation can also be found in Berres, Bürger \& Tory (2005). Sedimentation in a convecting fluid is studied in Lavorel \& Le Bars (2009).

\subsection{The dynamics of sedimentation and resuspension}

\subsubsection{Equilibrium profile}

Equation (2.14) describes the evolution of the particle volume fraction in the presence of bulk horizontal flow, together with internal sedimentation and resuspension. For a steady flow with no $x$ variation (2.14) reduces to a one-dimensional differential equation for $\alpha$. We introduce the Péclet number $P e \equiv V H / D$, defined as the ratio 
of sedimentation time scale $V / H$ and diffusion time scale $D / H^{2}$. The height of the flowing body, denoted by $H$, defines the observation scale. This yields

$$
\partial_{y}\left[P e \alpha\left(1-\frac{\alpha}{\phi}\right)+H \partial_{y} \alpha\right]=0 .
$$

By identifying $P e=1 / z_{0}$ the same equation can be found in Gray \& Chugunov (2006) as the equilibrium equation for the segregation-remixing problem. Since the flux must vanish at the upper and lower surfaces it must be zero everywhere. This implies that sedimentation $\operatorname{Pe} \alpha(1-\alpha / \phi)$ exactly balances the diffusion $H \partial_{y} \alpha$. The vertical distribution of the particles throughout the mixture is determined by this balance. Henceforth we will refer to the vertical distribution of $\alpha$ as the solids profile. A large Péclet number corresponds to a profile dominated by sedimentation, in which $\alpha$ will be close to $\phi$ in a region near the ground and close to 0 near the surface. In the small-Péclet-number case the diffusive flux dominates sedimentation, which implies an almost uniform mixture. For a density ratio equal to one $(\delta=1)$ we have $P e=0$, and hence $\partial_{y} \alpha=0$ which implies $\alpha=$ constant. The solution to (2.15) on the interval $y \in[0, H]$ is given by

$$
\alpha(y)=\phi \frac{\mathrm{e}^{-P e y / H}}{\mathrm{e}^{-P e y / H}-C} .
$$

$C$ is a positive constant of integration and is chosen in such a way that it fulfils the constraint $H_{p} \equiv \int_{0}^{H} \alpha(y) \mathrm{d} y . H_{p}$ denotes the $y$-integrated particle volume of the mixture, hence $H_{p} / H$ stands for the average volume fraction. For a constant $\alpha \equiv 0.5$ we for instance have $H_{p}=H / 2$. We find

$$
C=\frac{\exp \left[P e H_{p} / H-\phi / H \phi\right]-1}{\exp \left[P e\left(H_{p} / H\right) / H \phi\right]-1} .
$$

For an average volume fraction that equals the maximum packing fraction, hence $H_{p} / H=\phi$, the solution degenerates to $\alpha \equiv \phi$. Larger values for $H_{p} / H$ are not physical. Some specific solutions for the particle profile $\alpha(y)$ are displayed in figure 2 and resemble figure 3 of Gray \& Chugunov (2006).

\subsubsection{Relaxation towards the equilibrium}

In order to investigate how the system is forced into its equilibrium (2.16), we consider the unsteady sedimentation-resuspension process

$$
\partial_{t} \alpha=\partial_{y}\left[V \alpha\left(1-\frac{\alpha}{\phi}\right)+D \partial_{y} \alpha\right] .
$$

If $\alpha$ were scaled by $\phi$ this would be the same equation as presented by Gray \& Thornton (2005) and Gray \& Chugunov (2006) for segregation and remixing processes in bi-disperse granular media. We follow the same solution approach. The full initial value problem (IVP) is given by

$$
\begin{gathered}
0<y<H: \quad \partial_{t} \alpha-\partial_{y}\left[V \alpha(1-\alpha / \phi)+D \partial_{y} \alpha\right]=0, \\
t=0: \quad \alpha(0, y)=\alpha_{0}(y), \\
y=0, H: \quad V \alpha(1-\alpha / \phi)+D \partial_{y} \alpha=0 .
\end{gathered}
$$



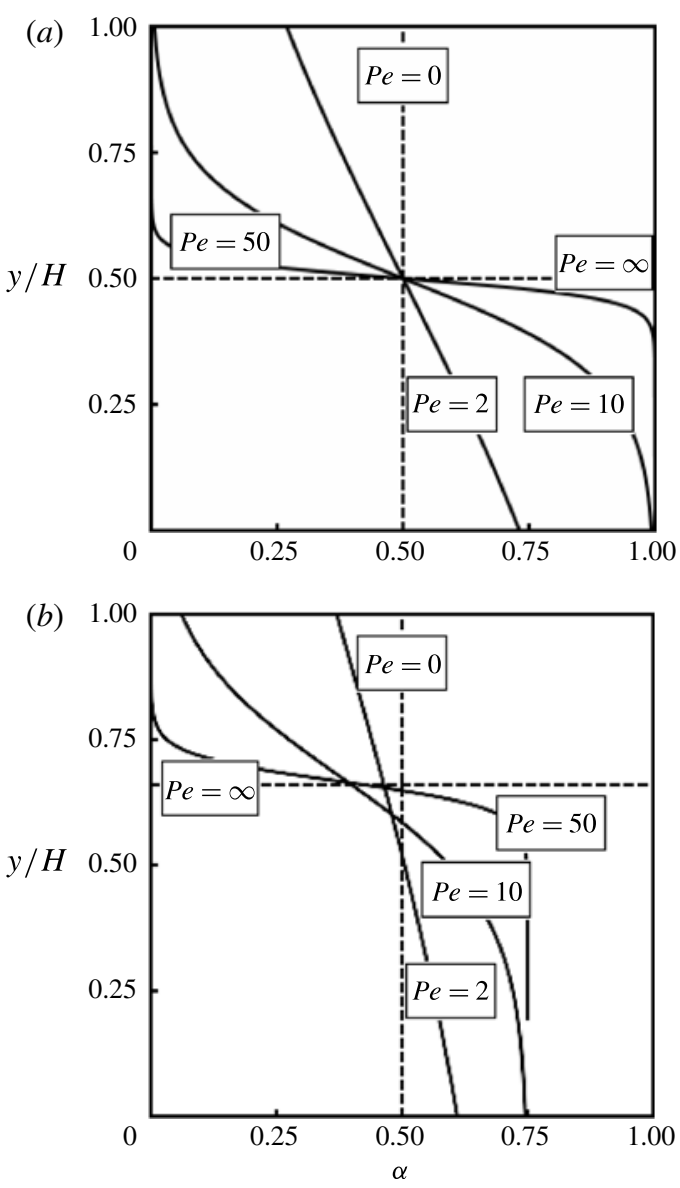

FIGURE 2. Equilibrium solution of the solids profile $\alpha(y)$ : $\alpha$ versus normalized height $y / H$. The integrated particle volume is $H_{p}=0.5$, and in $(a)$ the maximum packing fraction is $\phi=1.0$ and in $(b) \phi=0.75$. Large values of $P e$ correspond to a profile dominated by sedimentation, that is a lower region of maximum solids fraction and an upper region of pure fluid. Small values of $P e$ correspond to a well-mixed layer where the solids fraction only decreases slowly with depth. The two limiting states are given by $P e=\infty$ (completely settled) and $P e=0$ (homogeneous mixture).

We introduce space and time scaled coordinates, as well as $\psi$, a Cole-Hopf transformation of the volume fraction $\alpha$ :

$$
t=\frac{D \phi^{2}}{V^{2}} \tau, \quad z=\frac{D \phi}{V} \zeta, \quad \alpha=\frac{1}{2}\left(\phi-2 \frac{\partial_{\zeta} \psi}{\psi}\right) .
$$

Substituting these into the IVP yields a linear diffusion equation with linear boundary conditions:

$$
\begin{gathered}
0<\zeta<\zeta_{H}: \quad \partial_{\tau} \psi=\partial_{\zeta}^{2} \psi \\
\tau=0: \quad \psi_{0}(\zeta)=\exp \left(\int_{0}^{\zeta} \alpha_{0}\left(\zeta^{\prime}\right)-\frac{\phi}{2} \mathrm{~d} \zeta^{\prime}\right) \\
\zeta=0, \zeta_{H}: \quad \partial_{\zeta}^{2} \psi=\frac{\phi^{2}}{4} \psi .
\end{gathered}
$$


In order to simplify boundary condition (2.21c) we follow Gray \& Chugunov (2006) and separate out the exponentially decaying contribution $\mathrm{e}^{\beta \tau}$ by making the ansatz

$$
\psi(\zeta, \tau)=\mathrm{e}^{\beta \tau} \omega(\zeta, \tau),
$$

which yields

$$
\begin{gathered}
0<\zeta<\zeta_{H}: \quad \partial_{\tau} \omega=\partial_{\zeta}^{2} \omega-\beta \omega, \\
\tau=0: \quad \omega_{0}(\zeta)=\psi_{0}(\zeta), \\
\zeta=0, \zeta_{H}: \quad \partial_{\zeta}^{2} \omega=\frac{\phi^{2}}{4} \omega .
\end{gathered}
$$

At steady state $\partial_{\zeta}^{2} \omega_{s}=\beta \omega_{s}$, the boundary condition (2.23c) reduces to $\left(\beta-\phi^{2} / 4\right) \omega_{s}=0$. And, since $\omega_{s}$ does not vanish at the boundaries $\zeta=0, \zeta_{H}(2.21 c)$ can only hold if $\beta \equiv \phi^{2} / 4$. For this choice of $\beta$, however, we can substitute (2.23a) into $(2.23 c)$, and get $\partial_{\tau} \omega_{s}=0$. Since $\omega_{s}$ does not change in time it is completely determined by the initial condition according to

$$
\begin{aligned}
& \zeta=0: \quad \omega(0, \tau) \equiv 1 \\
& \zeta=\zeta_{H}: \quad \omega\left(\zeta_{H}, \tau\right) \equiv \psi_{0}\left(\zeta_{H}\right) .
\end{aligned}
$$

The solution to the linear diffusion equation can now be written as the superposition of a steady contribution $\omega_{s}$, and an unsteady part, $\omega_{u}$. Integration yields the steady part

$$
\omega_{s}(\zeta)=C_{1} \mathrm{e}^{-\zeta / 2}+C_{2} \mathrm{e}^{\zeta / 2}
$$

with $C_{1}=\left(\mathrm{e}^{\zeta_{H}}-\psi_{0}\left(\zeta_{H}\right) \mathrm{e}^{\zeta_{H} / 2}\right) /\left(\mathrm{e}^{\zeta_{H}}-1\right)$ and $C_{2}=1-C_{1}$. Back-transformation yields the equilibrium solution computed earlier. The unsteady part is solved in terms of Fourier modes and results in

$$
\omega_{u}(\tau, \zeta)=\sum_{n=1}^{\infty} a_{n} \mathrm{e}^{-\mu_{n}^{\prime} \tau} \sin \left(\frac{n \pi \zeta}{\zeta_{H}}\right),
$$

where $\mu_{n}^{\prime}=\left(n^{2} \pi^{2} / \zeta_{H}^{2}\right)+\left(\phi^{2} / 4\right)$ are the dimensionless decay constants. The Fourier sine coefficients $a_{n}$ are given by

$$
a_{n}=\frac{2}{\zeta_{H}} \int_{0}^{\zeta_{H}}\left[\psi_{0}(0, \zeta)-\omega_{s}(0, \zeta)\right] \sin \left(\frac{n \pi \zeta}{\zeta_{H}}\right) \mathrm{d} \zeta .
$$

All in all, we get a solution of the form

$$
\psi(\zeta, \tau)=\mathrm{e}^{\tau \phi^{2} / 2}\left(\omega_{s}(\zeta)+\omega_{u}(\zeta, \tau)\right)
$$

The corresponding expression for $\alpha$ is given by reversing the time and space scaling as well as the Cole-Hopf transformation in (2.20). Figure 3 is once again inspired by the previous work of Gray \& Chugunov (2006) and shows the solution to (2.18) for two different initial configurations, the first being a homogeneous mixture in the vertical direction and the second an inversely stratified initial condition. For more details on the derivation we refer to Gray \& Chugunov (2006). That paper also includes the analysis of various initial configurations.

\subsection{Projection on to the vertical centre of mass}

In order to incorporate information on the vertical profile into a depth-averaged theory, we will now project $\alpha(t, y)$ onto two scalar, time-dependent variables. The first of 

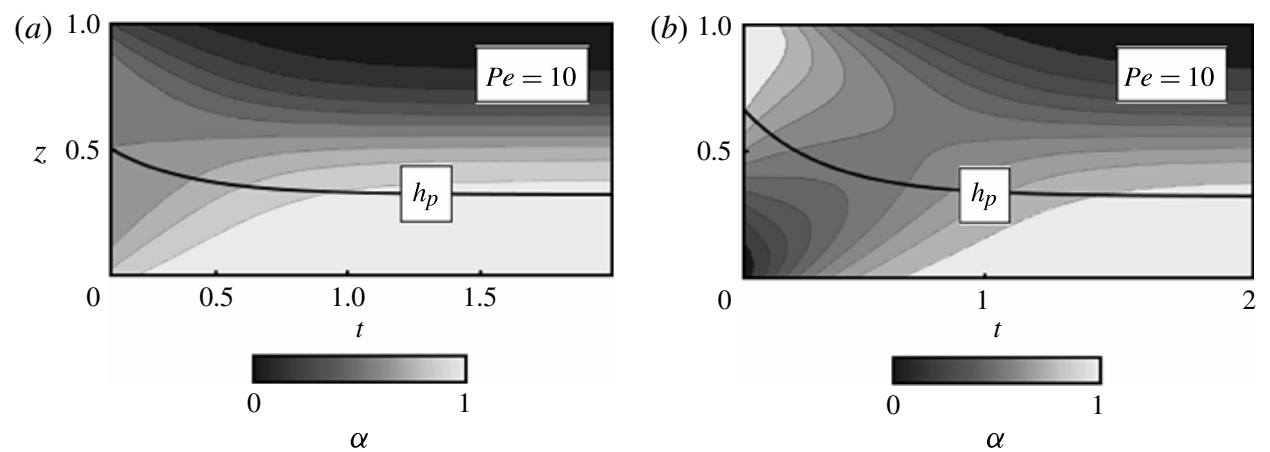

FIGURE 3. The evolution of a mixture's solids profile $\alpha(y)$ with time $t$ towards the equilibrium profile for $H_{p}=0.5, P e=10$ and $\phi=1$. The $y$-axis indicates the normalized depth of the flow and the greyscale the solids volume fraction $\alpha(y)$. For $P e=10$ sedimentation dominates diffusion and hence the particles tend to accumulate near the ground in equilibrium. The two plots differ in their initial conditions: $(a)$ an initially homogeneous mixture throughout the depth and $(b)$ an unstably stratified initial condition in which the particles are at the top. The thick line indicates the position of the vertical centre of mass $h_{p}$ for every point in time. In $(b)$ the relaxation time for the inversely stratified initial condition is slightly longer.

these is simply the equivalent height mass holdup $H_{p}$. A natural candidate for the second is the vertical centre of mass, defined as the ratio of the solids density first moment with respect to $y$ and the solids mass holdup,

$$
h_{p}(t) \equiv \frac{\int_{0}^{H} y \rho_{p}(y, t) \mathrm{d} y}{\int_{0}^{H} \rho_{p}(y, t) \mathrm{d} y}=\frac{\int_{0}^{H} y \alpha(y, t) \mathrm{d} y}{\int_{0}^{H} \alpha(y, t) \mathrm{d} y}=\frac{1}{H_{p}(t)} \int_{0}^{H} y \alpha(y, t) \mathrm{d} y,
$$

since $\rho_{p}=\alpha \varrho_{p}$. One motivation for this choice is that it captures the gravitational potential energy of the solid component. Also, for a non-constant vertical mixture density, it is exactly the mass centre $h_{p}$ that couples back into the hydrostatic pressure contribution to the momentum balance flux. Other advantages will become obvious later in the paper.

In order to evaluate the first moment of the vertical profile, we notice that the modes corresponding to $n>0$ are all decaying exponentially with decay rates proportional to $n^{2}$, that is rapidly increasing. For a system close to equilibrium it is therefore reasonable to keep only the first mode. This reduction to the normal manifold approach is standard in dynamical systems theory and also forms part of a rigorous derivation of the standard shallow-water equations. The solution with just the first mode is

$$
\omega(\tau, \zeta)=C_{1} \mathrm{e}^{-\zeta / 2}+C_{2} \mathrm{e}^{\zeta / 2}+a_{1} \mathrm{e}^{-\mu_{1}^{\prime} \tau} \sin \left(\frac{\pi \zeta}{\zeta_{H}}\right) .
$$

Inverting the Cole-Hopf transform gives an expression for $\alpha$,

$$
\alpha(t, y)=\frac{\phi}{2}-\frac{\phi D}{V} \frac{\mathrm{d}}{\mathrm{d} y} \log \left[\omega_{s}(y)+A_{1}(t) \sin \left(\frac{\pi y}{H}\right)\right],
$$


in which $A_{1}(t) \equiv a_{1} \mathrm{e}^{-\mu_{1}^{\prime} \tau}=a_{1} \mathrm{e}^{-\mu_{1} t}$, where $\mu_{1}=\left(\pi^{2} D / H^{2}\right)+\left(V^{2} / 4 D\right)$. We get

$$
h_{p}(t)=\frac{1}{H_{p}} \int_{0}^{H} y\left(\frac{\phi}{2}-\frac{\phi D}{V} \frac{\mathrm{d}}{\mathrm{d} y} \log \left[\omega_{s}(y)+A_{1}(t) \sin \left(\frac{\pi y}{H}\right)\right] \mathrm{d} y\right) .
$$

Now $A_{1}(t)$ satisfies the differential equation

$$
\frac{\mathrm{d} A_{1}}{\mathrm{~d} t}=-\mu_{1} A_{1} .
$$

Using (2.32) we can regard $h_{p}$ as an invertible function of $A_{1}$. Therefore we have

$$
\frac{\mathrm{d} h_{p}}{\mathrm{~d} t}=\frac{\mathrm{d} h_{p}}{\mathrm{~d} A_{1}} \frac{\mathrm{d} A_{1}}{\mathrm{~d} t}=-\mu_{1} A_{1}\left(h_{p}\right) \frac{\mathrm{d} h_{p}}{\mathrm{~d} A_{1}} .
$$

Thus if we can invert (2.32) and calculate the integral defining $\mathrm{d} h_{p} / \mathrm{d} A_{1}$ we know the exact evolution equation for $h_{p}$. This cannot be done in terms of simple functions; however it could be straightforwardly implemented in a numerical code. We will pursue an analytic approach in this paper by expanding $h_{p}$ about its equilibrium value $h_{p}^{(s)}=\left(1 / H_{p}\right) \int_{0}^{H} y \alpha^{(s)}(y) \mathrm{d} y$, which is given by a dilog function. Since $h_{p}=h_{p}^{(s)}$ when $A_{1}=0$ to lowest order we get

$$
\frac{\mathrm{d}}{\mathrm{d} t} h_{p}=\mu_{1}\left(h_{p}^{(s)}-h_{p}\right)
$$

That is the evolution of the solids centre of mass $h_{p}$ is determined by a relaxation towards equilibrium $h_{p}^{(s)}$ with a decay rate given by the first Fourier mode $\mu_{1}$. The accuracy of this equation can easily be improved by expanding (2.34) in higher powers of $h_{p}^{(s)}-h_{p}$. In figure 3 the time evolution of the solution to the unsteady sedimentation-resuspension equation is shown along with the evolution of the vertical mass centre as a thick curve.

The diffusion in the flow is primarily due to turbulent eddies and hence scales with both the typical eddy size $H^{2}$ and the local shear rate $U / H, U$ being a characteristic horizontal velocity. Thus we expect $D \propto H U=\bar{D} H U$, in which $\bar{D}$ is a dimensionless proportionality coefficient. This choice yields

$$
\frac{\mathrm{d}}{\mathrm{d} t} h_{p}=\frac{V}{H}\left(\pi^{2} \frac{\bar{D} U}{V}+\frac{1}{4} \frac{V}{\bar{D} U}\right)\left(h_{p}^{(s)}-h_{p}\right)
$$

Written like this the primary time scale is given by $H / V$, the sedimentation through the depth. If this is kept constant and $\bar{D} U / V$ allowed to vary then there is a minimum decay rate when $V / \bar{D} U=2 \pi$ of $\mu_{1}=\pi(V / H)$.

\subsection{Resuspension-dominated flow regime}

When the sedimentation velocity is small compared to the bulk horizontal velocity $U$, the decay rate of (2.35) can be approximated by

$$
\mu_{1}=\pi^{2} \bar{D} \frac{U}{H},
$$

which scales linearly with a first-order approximation to the shear rate. In this flow regime the diffusion flux forces the mixture into a well-mixed flow state, hence $h_{p}^{(s)} \approx H / 2$. Consequently, the mass centre $h_{p}$ evolves according to

$$
h_{p}(t)=\frac{H}{2}+\left(\frac{H_{p}}{2 \phi}-\frac{H}{2}\right) \exp \left[-\pi^{2} t \bar{D} \frac{U}{H}\right] .
$$




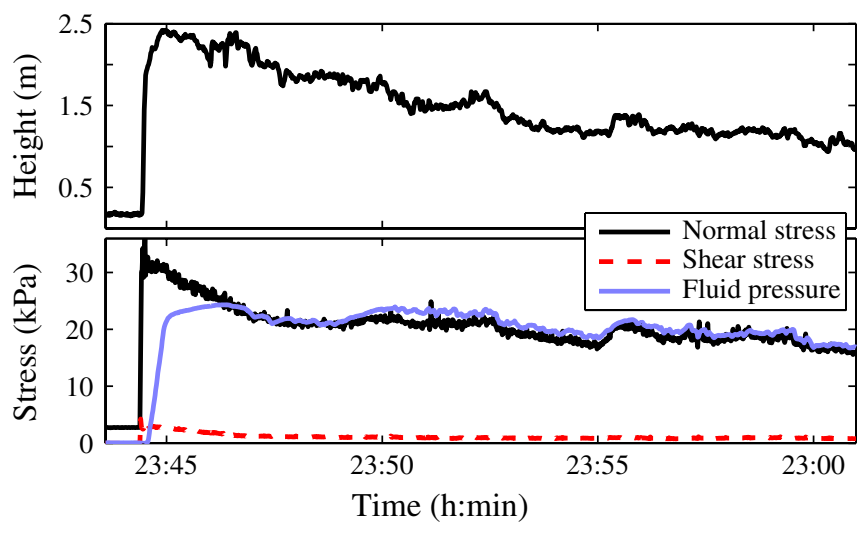

FIGURE 4. (Colour online) Large-scale data of a debris flow event in the Illgraben torrent, Switzerland on 28 May 2005. Height, normal stress and basal fluid pressure are measured and plotted versus time.

We speak of a resuspension-dominated regime, a flow state that may for instance be present in the initiation phase of a multi-component geophysical mass flow. Particles are eroded from the ground and resuspension fluxes force them into a well-mixed suspension. The vertical mass centre is adjusted to the presence of 'new' particles with a time delay accounted for by the relaxation time.

Figure 4 shows the initial $15 \mathrm{~min}$ period of a large natural debris flow event in the Swiss Alps. The main flow lasted for another hour. The entire flow transported a bulk mixture volume of around $140000 \mathrm{~m}^{3}$. The plots show measured surface height, total normal stress and effective basal fluid pressure at one specified point in the flow channel over time. The height data clearly indicate the arrival of the front, and the same abrupt increase is seen in the normal stress. The basal fluid pore pressure however, which can be understood as a measure of particles in suspension shows a lag. The proposed model explains this lag as the time required to fully suspend the particles eroded in the head of the flow. In the first seconds after the arrival of the front the lower relative level of the pore pressure may of course also be due to the fact that the flow is not fully saturated. Visual observations, however, indicate that the unsaturated part only lasts for a couple of seconds, whereas the adjustment of the pore pressure level is of the order of minutes. Full suspension is being indicated by the fact that the pore pressure measurement coincides with the normal stress measurement, as is seen in the tail of the flow.

\subsection{Sedimentation-dominated regime}

When the horizontal bulk velocity $U$ is small the system will be dominated by sedimentation and diffusion/resuspension will be small. The flow may no longer be turbulent and the scalings of (2.36) will be invalid. The time scale should be proportional to $H / V$, the sedimentation time, and we note that if this is kept constant and $V / \bar{D} U$ varied the minimum value of the decay rate is

$$
\mu_{1}=\pi \frac{V}{H} .
$$

$V$ will usually be very small such that relaxation of the vertical mass centre due to sedimentation will happen on a much longer time scale than for the resuspension 
process. In terms of geophysical mass flows this, among other flow situations, refers to the deposition zone in which horizontal velocities are negligible. Within a single vertical column of the mixture the individual masses are conserved and only the solids vertical centre of mass $h_{p}$ is subject to change. The equilibrium solids mass centre $h_{p}^{(s)}$ is evaluated to be

$$
h_{p}^{(s)}=\frac{H_{p}}{2 \phi} .
$$

If initially the particles are distributed uniformly throughout the height, sedimentation is given by exponential decay towards equilibrium, hence

$$
h_{p}(t)=\frac{H_{p}}{2 \phi}+\left(\frac{H}{2}-\frac{H_{p}}{2 \phi}\right) \mathrm{e}^{-\pi t V / H} .
$$

\subsection{Connection to the basal fluid pressure}

In recent experiments the effective basal fluid pressure of real-scale and laboratoryscale debris flows has been measured (Iverson \& Major 1999; McArdell, Bartelt \& Kowalski 2007). By effective basal fluid pressure we mean the apparent fluid pressure measured at the base of the flow $\left.p_{f}\right|_{y=0}=\left.p_{h}\right|_{y=0}+\left.p_{e}\right|_{y=0}$. It is also referred to as the fluid pore pressure. Now from from (2.11) we have $p_{e}=X \bar{p}$ and on the basal surface $\bar{p}=\left(\varrho_{p}-\varrho_{f}\right) g_{y} H_{p}$ and $p_{h}=\varrho_{f} g_{y} H$, thus

$$
\begin{aligned}
\left.p_{f}\right|_{y=0} & =\varrho_{f} g_{y} H+X_{y=0}\left(\varrho_{p}-\varrho_{f}\right) g_{y} H_{p} \\
& =g_{y} H\left[\varrho_{f}+\frac{H_{p}}{H}\left(1-\frac{\alpha_{y=0}}{\phi}\right)\left(\varrho_{p}-\varrho_{f}\right)\right] .
\end{aligned}
$$

We can see clearly how the solids volume fraction near the basal surface $\left(\alpha_{y=0}\right)$ determines the fluid pressure. If the solids concentration is low then the particles are entirely suspended and the fluid pressure is $g_{y}\left[\left(H-H_{p}\right) \varrho_{f}+H_{p} \varrho_{p}\right]$, the weight of all the particles and all the water. If the particles are at their maximum steady concentration $\phi$ then the fluid pressure is $g_{y} H \varrho_{f}$ corresponding to a column of pure water. If the particles are over-compressed $\alpha>\phi$ then the fluid pressure can be less than this. This is the case initially in a flow as the grains have to dilate before they can move and such negative pore pressures have been seen in experiments (Pailha \& Pouliquen 2008).

To calculate the fluid pressure in terms of $h_{p}$ it is necessary to invert (2.32) to calculate $A_{1}$ and then to evaluate (2.31) at $y=0$. In a numerical code this can be done, but for illustrative purposes we again work analytically and expand about the equilibrium state. From (2.31) we have

$$
A_{1}=\lambda\left(h_{p}-h_{p}^{(s)}\right)
$$

where

$$
\lambda=\frac{\phi}{H_{p}} \int_{0}^{H}\left[\frac{\pi D}{V H} \frac{\cos (\pi y / H)}{\omega_{s}(y)}+\frac{1}{2} \frac{\sin (\pi y / H)}{\omega_{s}(y)^{2}}\left(C_{1} \mathrm{e}^{-V y / D}-C_{2} \mathrm{e}^{V y / D}\right)\right] y \mathrm{~d} y .
$$

Now

$$
\begin{aligned}
\alpha_{y=0} & =\phi \frac{1-\mathrm{e}^{-V H_{p} / \phi D}}{1-\mathrm{e}^{-V H / \phi D}}-\frac{\pi \phi D A_{1}}{V H} \\
& =\phi \frac{1-\mathrm{e}^{-V H_{p} / \phi D}}{1-\mathrm{e}^{-V H / \phi D}}-\frac{\pi \phi D \lambda}{V H}\left(h_{p}-h_{p}^{(s)}\right) .
\end{aligned}
$$


Note that if $H_{p}=\phi H$ then $\lambda=0$ and $\alpha_{y=0}=\phi$ corresponding to a saturated collection of particles at maximum packing. This then can be substituted into (2.42) to give the fluid pressure on the base

$$
\begin{aligned}
\left.p_{f}\right|_{y=0} & =\varrho_{f} g_{y} H+X_{y=0}\left(\varrho_{p}-\varrho_{f}\right) g_{y} H_{p} \\
& =g_{y} H\left[\varrho_{f}+\frac{H_{p}}{H}\left(\frac{\mathrm{e}^{-V H_{p} / \phi D}-\mathrm{e}^{-V H / \phi D}}{1-\mathrm{e}^{-V H / \phi D}}+\frac{\pi D \lambda}{V H}\left(h_{p}-h_{p}^{(s)}\right)\right)\left(\varrho_{p}-\varrho_{f}\right)\right] .
\end{aligned}
$$

Though this expression is rather complicated, especially with the definition of $\lambda$, the physics it describes are straightforward. In equilibrium $\left(h_{p}-h_{p}^{(s)}\right)$ there is an excess pore pressure that is governed by the volume fraction of solid particles $H_{p} / H$ and the Péclet number $V H / D$. If the centre of mass of the particles is higher than equilibrium, so that there is net sedimentation, then the pore pressure is increased. If the centre of mass of the particles is lower than equilibrium, so that there is net resuspension, then the pore pressure is reduced.

\section{Depth-averaging}

Depth-averaging refers to an integration along the vertical (slope-normal) $y$-axis. It is an appropriate tool to reduce a problem's dimensionality whenever the shallowness parameter, given by the ratio of characteristic height and characteristic length, is small. The formulation of geophysical mass flows in terms of depth-averaged models is very common and has been rigorously derived and analysed for all kinds of one-phase flows (see Savage \& Hutter 1989, 1991; Bartelt, Salm \& Gruber 1999; Wieland, Gray \& Hutter 1999; Gray 2002; Pudasaini \& Hutter 2007). There also exist some generalizations to multi-component mixtures (see Iverson 1997; Iverson \& Denlinger 2001; Pitman \& Le 2005). A depth-averaged formulation of the segregation model proposed in Gray \& Chugunov (2006) is given in Gray \& Kokelaar (2010).

In this section we present a depth-averaged shallow flow model in which the balance laws for mass and momentum are extended by a depth-averaged concentration equation. This itself is not new and in a slightly different formulation has already been proposed by Pitman \& Le (2005). The innovation, however, comes from allowing for a non-constant mixture density in the vertical direction, which translates into a coupling of the momentum balance to the vertical centre of mass. The model is therefore completed by an evolution equation for the vertical mass centre, derived by depth-averaging the first moment of the concentration equation.

\subsection{Depth-averaged quantities}

We assume that the flow has a clearly defined free surface $y=H(x, t)$, also referred to as the height of the flow. Integration of the densities $\rho, \rho_{p}$ and $\rho_{f}$ over the vertical $y$-axis results in bulk mass per unit area $m$ as well as the masses of the individual components $m_{p}$ and $m_{f}$ per unit area. We have

$$
m \equiv \int_{0}^{H} \rho \mathrm{d} y, \quad m_{i} \equiv \int_{0}^{H} \rho_{i} \mathrm{~d} y,
$$

with $i \in\{f, p\}$. For a constant mixture density $\rho$ we have $m=H \rho$ in which case $H$ recovers the flow height commonly used as a system variable in depthaveraged single-component theories (Savage \& Hutter 1989, 1991; Bartelt et al. 1999; Wieland et al. 1999; Gray 2002; Pudasaini \& Hutter 2007). We also want to depthaverage stresses and velocities. For the integrals to be well-defined we introduce the 
density-weighted-average notation

$$
\langle\cdot\rangle=\frac{\int_{0}^{H}(\cdot) \rho \mathrm{d} y}{\int_{0}^{H} \rho \mathrm{d} y} \text { and }\langle\cdot\rangle_{i}=\frac{\int_{0}^{H}(\cdot) \rho_{i} \mathrm{~d} y}{\int_{0}^{H} \rho_{i} \mathrm{~d} y} .
$$

We define the depth-averaged particles mass concentration $c \equiv\left\langle\rho_{p} / \rho\right\rangle$ which relates to bulk mass and solids mass according to $m_{p}=\mathrm{cm}$. Depth-integration of the equation of state (2.3) yields an algebraic relation for the surface flow height

$$
H=H_{p}+H_{f}=\frac{m_{p}}{\varrho_{p}}+\frac{m_{f}}{\varrho_{f}}=\left(c+\frac{1-c}{\delta}\right) \frac{m}{\varrho_{p}} .
$$

In fact, any two variables of the set $\left\{m_{f}, m_{p}, m, c\right\}$ constitute an independent pair that uniquely describes the varying composition of the flow. The vertical volume average is given by $H_{p}=m_{p} / \varrho_{p}$ and accordingly we have $H_{f}=m_{f} / \varrho_{f}$. The depth-averaged barycentric velocity is denoted by $U \equiv\langle u\rangle$.

The essential idea of the proposed model generalization is the additional consideration of the bulk vertical centre of mass $h$ and the corresponding individual mass centres $h_{i}, i \in\{f, p\}$ as system variables. They are defined according to

$$
h \equiv\langle y\rangle, \quad h_{f} \equiv\langle y\rangle_{f} \quad \text { and } \quad h_{p} \equiv\langle y\rangle_{p},
$$

and contain information about the vertical structure of the flowing body. For a vanishing Péclet number $(P e=0)$ the equilibrium profile is given by a uniform mixture throughout the height. In that case, the mass centre of each component coincides with the mass centre of the bulk at half the surface height. We have $h=h_{f}=h_{p}=H / 2$.

In order to see how the vertical mass centre relates to the depth-averaged masses and volumes, we integrate volume and density constraints multiplied by $y$,

$$
y \rho=y \rho_{p}+y \rho_{f} \quad \text { and } \quad y=y \alpha+y(1-\alpha),
$$

which results in

$$
h m=h_{p} m_{p}+h_{f} m_{f} \quad \text { and } \quad \frac{H^{2}}{2}=h_{p} H_{p}+h_{f} H_{f} .
$$

This makes explicit that there is only one degree of freedom in the set $\left\{h, h_{f}, h_{p}\right\}$ and knowing one mass centre already implies the value of the other two. We will formulate the equations in terms of the particle centre of mass $h_{p}$. Solving for $h$ then yields

$$
h=\frac{\varrho_{f}}{m}\left(\frac{H^{2}}{2}-\left(1-\frac{1}{\delta}\right) H_{p} h_{p}\right) .
$$

For $\delta=1$ this reduces to $h=H / 2$.

\subsection{Velocity shear and particle profile}

There is a coupling between vertical variation of the barycentric velocity and particle concentration that introduces additional fluxes. Calculating the coupling explicitly for general solid profiles like the ones derived in the previous sections results in rather complicated expressions and needs numerical approximations. To highlight the mechanism, however, we consider the limit of small Péclet numbers, hence a 
resuspension-dominated regime, in which a linear solid particle profile can be assumed. We define

$$
\alpha(y)=\frac{H_{p}}{H}\left(1+12\left(\frac{y}{H}-\frac{1}{2}\right) h_{p}^{\prime}\right),
$$

in which $h_{p}^{\prime} \equiv\left(h_{p} / H\right)-(1 / 2)$, and a longitudinal velocity profile with a linear dependence in $y$ given by

$$
u(y)=U+U \gamma\left(\frac{y}{H}-\frac{1}{2}-\delta c h_{p}^{\prime}\right) .
$$

The definitions for $\alpha(y)$ and $u(y)$ are consistent with the definition of $m, c, U$ and $h_{p}$. Here, $\gamma$ is a non-dimensional measure of shear. If we choose $1 / \gamma=(1 / 2)+c \delta h_{p}^{\prime}$, this corresponds to a non-slip basal boundary condition. For a fully developed turbulent current $\gamma=0$ is a good choice. A more developed theory could consider a separate evolution equation for $\gamma$ or an algebraic closure related to mass entrainment on the lower surface.

The resulting fluxes in the depth-averaged setting are evaluated analytically and demonstrate the form of the additional terms that might appear in a model.

\subsection{A depth-averaged flux hierarchy}

The local quantity $(\cdot)$, the depth-integrated quantity $\int_{0}^{H}(\cdot) \mathrm{d} y$ and the corresponding horizontal flux $\int_{0}^{H}(\cdot) u \mathrm{~d} y$ are given by the following triples:

$$
\begin{array}{ccl}
\text { local field } & \text { depth-averaged quantity } & \multicolumn{1}{c}{\text { flux }} \\
(\cdot) & \int_{0}^{H}(\cdot) \mathrm{d} y & \int_{0}^{H}(\cdot) u \mathrm{~d} y \\
1 & H & H U\left(1-\gamma \delta c h_{p}^{\prime}\right) \\
\rho & m & m U \\
\rho_{p} & c m & c m U\left(1+\gamma(1-\delta c) h_{p}^{\prime}\right) \\
y \rho_{p} & c m h_{p} & c m h_{p} U\left(1+\gamma\left(\frac{1}{2}-\delta c h_{p}^{\prime}-\frac{1}{6} \frac{H}{h_{p}}\right)\right) \\
\rho u & m u & m U^{2}\left(1+\gamma^{2}\left(\frac{1}{12}-\delta^{2} c^{2} h_{p}^{\prime 2}\right)\right)
\end{array}
$$

\subsection{Depth-averaged mass conservation}

Integration of the bulk mass conservation (2.2a) and the solids mass conservation (2.14) yields

$$
\begin{gathered}
\int_{0}^{H}\left(\partial_{t} \rho+\partial_{x}(\rho u)\right) \mathrm{d} y=-[\rho w]_{0}^{H}, \\
\int_{0}^{H}\left(\partial_{t} \rho_{p}+\partial_{x}\left(\rho u_{p}\right)\right) \mathrm{d} y=-\left[\rho_{p} w\right]_{0}^{H}+\varrho_{p}\left[\frac{V}{\phi} \alpha(\phi-\alpha)+D \partial_{y} \alpha\right]_{0}^{H} .
\end{gathered}
$$

We assume zero mass flux at the material boundaries, such that $\rho w$ and $\rho_{p} w$ evaluated at the free surface and the bottom are zero $\left(\left.\rho w\right|_{y=0, H}=\left.\rho_{p} w\right|_{y=0, H}=0\right)$. There is another contribution to the right-hand side of the concentration equation $(3.11 b)$, which evaluates sedimentation and resuspension fluxes at the boundaries. This term also 
vanishes due to zero flux boundary conditions $(2.19 \mathrm{c})$. Identifying $\mathrm{m}$ and $\mathrm{cm}$ and substituting for the fluxes yields

$$
\begin{gathered}
\partial_{t} m+\partial_{x}(m U)=0, \\
\partial_{t}(c m)+\partial_{x}\left[c m U\left(1+\gamma(1-\delta c) h_{p}^{\prime}\right)\right]=0 .
\end{gathered}
$$

By expanding the derivatives and subtracting off $c$ times (3.12a) this can be written as a transport equation for the depth-averaged concentration $c$ :

$$
\partial_{t} c+U \partial_{x} c+\frac{1}{m} \partial_{x}\left[c m U \gamma(1-\delta c) h_{p}^{\prime}\right]=0 .
$$

Bulk as well as individual masses are conserved when being advected with the depthaveraged barycentric velocity $U$. Quantities such as the average particle concentration $c$ do not obey pure conservation equations but contain source terms.

Assuming that the flow is fully saturated, that is $v=1$ for $0<y<H$ and $v=0$ elsewhere, and integration over $y$ yields an evolution equation for the surface height:

$$
\partial_{t} H+\partial_{x}\left[H U\left(1-\gamma \delta c h_{p}^{\prime}\right)\right]=[w]_{0}^{H}=0 .
$$

\subsection{Depth-averaged momentum balance}

Now we consider the depth-integrated horizontal momentum balance

$$
\int_{0}^{H}\left(\partial_{t}(\rho U)+\partial_{x}\left(\rho u^{2}\right)\right) \mathrm{d} y=-[\rho u w]_{0}^{H}+\int_{0}^{H}(-\nabla \sigma+\rho \boldsymbol{g}) \mathrm{d} y .
$$

A zero mass flux at the material boundaries once again implies that the bracketed term $[\rho u w]_{0}^{H}$ vanishes. In order to close for the stress tensor $\sigma$, we assume that internal deviatoric stresses are unimportant and are only relevant at the material boundaries. Locally the flow is in hydrostatic balance. The stress tensor then reduces to a heightdependent scalar given by

$$
\partial_{y} \sigma=-g_{y} \rho \Rightarrow \sigma(y)=g_{y} \int_{y}^{H} \rho \mathrm{d} y .
$$

This is a common assumption in geophysical shallow flow theory and does not differ from other approaches (see for instance Savage \& Hutter 1989, 1991; Bartelt et al. 1999; Wieland et al. 1999; Gray 2002; Pudasaini \& Hutter 2007). Usually the density is considered constant in $y$ which allows the direct evaluation $g_{y} \int_{y}^{H} \rho \mathrm{d} y=g_{y} H \rho$. We will, however, allow for a variable $\rho$ that may vary with height and continue with expression (3.16). The depth-averaged stress tensor is then given by

$$
\int_{0}^{H} \partial_{x} \sigma \mathrm{d} y+\left[\sigma_{x y}\right]_{0}^{H}=g_{y} \partial_{x} \int_{0}^{H} \int_{y^{\prime}}^{H} \rho \mathrm{d} y^{\prime} \mathrm{d} y+\left.\sigma_{x y}\right|_{y=0}=g_{y} h m+\left.\sigma_{x y}\right|_{y=0},
$$

$h$ being the vertical mass centre. The term $g_{y} h m$ stands for the hydrostatic pressure contribution to the momentum flux. For a constant $\rho$ we have $g_{y} h m=g_{y} \rho H^{2} / 2$, which is the well-known hydrostatic pressure forcing in a single-component shallow flow model. The term $\left.\sigma_{x y}\right|_{y=0}$ accounts for the basal shear stress and has to be closed in terms of a specific basal friction relation. In the original contribution of Savage \& Hutter (1989), a friction relation proportional to the normal stress is derived directly from a Mohr-Coulomb material assumption and results in dry Coulomb friction proportional to the normal force. In realistic applications usually Coulomb friction 
is superposed by a velocity-dependent contribution (see for instance Bartelt et al. 1999; McArdell et al. 2003; Cassar, Nicolas \& Pouliquen 2005; Christen et al. 2010).

For the sake of simplicity we will also use a simple Coulomb friction relation, hence $\left.\sigma_{x y}\right|_{y=0}=\mu g_{y} m, \mu$ being the friction coefficient, though more complicated approaches can easily be incorporated. In particular a more realistic model would include a dependence of the shear stress on the solid fraction at the base.

There is no contribution from the free surface as we assume a no-stress condition. Once again we identify $m$ and substitute for the fluxes:

$$
\partial_{t}(U m)+\partial_{x}\left[m U^{2}\left(1+\gamma^{2}\left(\frac{1}{12}-\delta^{2} c^{2} h_{p}^{2}\right)\right)+g_{y} h m\right]=\left(g_{x}-\mu g_{y}\right) m ;
$$

$\left\langle u^{2}\right\rangle / U^{2}=\gamma^{2}\left(1 / 12-\delta^{2} c^{2} h_{p}^{\prime 2}\right)$ is a shape factor that accounts for the deviation of the velocity profile (3.9) from plug flow $(\gamma=0)$. More realistic power-law profiles satisfying a no-slip condition, i.e. of the form $u(y)=u_{\text {surface }}\left\{1-[1-(y / H)]^{q}\right\}$ with $q>0$, imply $\left\langle u^{2}\right\rangle / U^{2}=(q+1)^{2} /(2 q+1)$. This is always greater than or equal to one with the lower bound attained for a plug flow in the limit $q=0$.

\subsection{A depth-averaged sedimentation-resuspension equation}

In order to track the vertical mass centre $h_{p}$, we derive an evolution equation by computing the first moment of the sedimentation-resuspension equation(2.14):

$$
\int_{0}^{H}\left[\partial_{t}\left(y \rho_{p}\right)+\partial_{x}\left(y \rho_{p} u\right)+y \partial_{y}\left(\rho_{p} w\right)\right] \mathrm{d} y=\int_{0}^{H} y \partial_{y}\left[\varrho_{p} V \alpha(1-\alpha / \phi)+D \partial_{y} \alpha\right] \mathrm{d} y .
$$

There are two dynamic processes which have to be considered: the barycentric horizontal advection of the bulk given by the flux on the left-hand side and the internal redistribution of solids within the fluid which acts as a source term on the right-hand side of the equation. Applying integration by parts transforms the left-hand side into

$$
\int_{0}^{H} \partial_{t}\left(y \rho_{p}\right)+\partial_{x}\left(y \rho_{p} u\right) \mathrm{d} y+\left[y \rho_{p} w\right]_{0}^{H}-\int_{0}^{H} \rho_{p} w \mathrm{~d} y .
$$

The boundary fluxes vanish $\left[y \rho_{p} w\right]_{0}^{H}=0$. Identifying $h_{p}$ yields

$$
\partial_{t}\left(c m h_{p}\right)+\partial_{x}\left(\operatorname{cmh}_{p} U\left(1+\gamma\left(\frac{1}{2}-\delta c h_{p}^{\prime}-\frac{1}{6} \frac{H}{h_{p}}\right)\right)\right)=\int_{0}^{H} \rho_{p} w \mathrm{~d} y .
$$

The vertical velocity at the free surface is given in terms of the kinematic condition $\left.w\right|_{y=H} \equiv \partial_{t} H+U \partial_{x} H$, whereas it is zero at the bottom surface. We therefore close $w$ according to

$$
w=\frac{y}{H}\left(\partial_{t} H+U \partial_{x} H\right) .
$$

By substituting (3.14) we can evaluate $\int_{0}^{H} \rho_{p} w \mathrm{~d} y=c m h_{p} \partial_{x} U$ and get

$$
c m\left(\partial_{t} h_{p}+\partial_{x}\left(h_{p} U\right)\right)+\partial_{x}\left(c m U H \gamma\left(\frac{1}{12}-h_{p}^{\prime 2}\right)\right) .
$$

The right-hand side of (3.19) accounts for a change in $h_{p}$ due to diffusion and resuspension and can likewise be simplified by applying integration by parts. Once again the boundary flux vanishes and we get

$$
\int_{0}^{H} y \varrho_{p} \partial_{y}\left(V \alpha\left(1-\frac{\alpha}{\phi}\right)+D \partial_{y} \alpha\right) \mathrm{d} y=-\int_{0}^{H} \varrho_{p}\left(V \alpha\left(1-\frac{\alpha}{\phi}\right)+D \partial_{y} \alpha\right) \mathrm{d} y .
$$


The response of vertical mass centre to sedimentation and resuspension has been rigorously analysed in the last section. According to (2.36) we can write

$$
-\int_{0}^{H} \varrho_{p}\left(V \alpha\left(1-\frac{\alpha}{\phi}\right)+D \partial_{y} \alpha\right) \mathrm{d} y=\mu_{1} c m\left(h_{p}^{(s)}-h_{p}\right) .
$$

This yields the final evolution equation for the vertical mass centre:

$$
c m\left[\partial_{t} h_{p}+\partial_{x}\left(h_{p} U\right)\right]+\partial_{x}\left[c m U H \gamma\left(\frac{1}{12}-h_{p}^{\prime 2}\right)\right]=\mu_{1} c m\left(h_{p}^{(s)}-h_{p}\right) .
$$

\section{An extended shallow flow model for fluid-particle mixtures}

In the last section we derived a set of depth-averaged balance laws for bulk mass, depth-averaged particle concentration, bulk momentum and vertical centre of mass. Following several authors (Iverson \& Denlinger 2001; Pitman \& Le 2005; Pelanti, Bouchut \& Mangeney 2008; Iverson 2009), we started from the balance laws for mass and momentum for both constituents, namely interstitial fluid and particles. Pitman \& Le (2005) integrated mass and momentum equations for the two species individually and arrived at a set of two depth-averaged mass conservation equations and two depth-averaged momentum balances. However, they found that their system became ill-posed for high slip velocities because of an elliptic degeneracy in their flux function. Pelanti et al. (2008) proposed a modification of the Pitman \& Le model that accounts for bulk momentum conservation. The model breakdown for high slip velocities is, however, also observed in their model.

In order to avoid these problems we followed the small-discharge scaling argument of Iverson (1997) and assumed the slip velocity to be small. We considered the mixture's barycentric velocity field and introduced relative rearrangement within the flowing body as a phase diffusion. Instead of the 'full' system of two depth-averaged momentum equations, we therefore arrive at the single depth-averaged momentum equation (3.18) and an algebraic equation for the slip velocity. Although the model is now restricted to cases of small slip velocities between the constituents, we will see in the rest of this section that is has the clear advantage of being hyperbolic within the physical domain of positive masses. Hence it is well-posed. A similar approach has been followed previously by Gray \& Kokelaar (2010) and led to a depth-integrated segregation model.

A new aspect of our approach is the consideration of varying fluid pressure in the direction depending on the solids concentration. In order to allow for a general vertical stress state we had to introduce yet another unknown to the system, the hydrostatic pressure contribution to the momentum balance. This term turns out to have a simple interpretation and is nothing but the vertical centre of mass. A closure is formulated by interpreting the vertical mass centre as the first density moment with respect to depthintegration. Separating out the bulk mass equation, momentum equation and vertical centre of mass equation and assuming the concentration to be constant, the proposed model resembles the general structure of Iverson's model (see for instance Iverson \& Denlinger 2001), in which depth-averaged bulk mass and momentum equations have been supplemented by a phenomenological evolution equation for the basal (fluid) pore pressure. A more recent publication is devoted to the derivation of the pore water evolution equation (Iverson 2009). 


\subsection{Mathematical structure and characteristic speeds}

In order to analyse the mathematical structure of the proposed model, we will set $\gamma \equiv 0$ and therefore assume a shape factor corresponding to plug flow $\left\langle u^{2}\right\rangle / U^{2} \equiv 1$. The extended fluid-solid shallow flow model $(3.12 a, b)$ and (3.18) then forms a set of three balance laws for the depth-averaged quantities $m, c$ and $U$. The equations contain the additional variable $h$, the vertical centre of mass. A closure is given by the evolution of the solids vertical centre of mass (3.26) together with the algebraic coupling (3.7). The summarized one-dimensional model then is written as

$$
\begin{gathered}
\partial_{t}\left(\begin{array}{c}
m \\
c m \\
m U \\
h_{p}
\end{array}\right)+\partial_{x}\left(\begin{array}{c}
m U \\
c m U \\
m U^{2}+g_{y} h m \\
h_{p} U
\end{array}\right)=\left(\begin{array}{c}
0 \\
0 \\
\left(g_{x}-\mu g_{y}\right) m \\
\mu_{1}\left(h_{p}^{(s)}-h_{p}\right)
\end{array}\right), \\
h=\frac{\varrho_{f}}{m}\left(\frac{H(c, m)^{2}}{2}-\left(1-\frac{1}{\delta}\right) H_{p}(c, m) h_{p}\right) .
\end{gathered}
$$

The mean definitions of the variables are summarized in (3.10) and table 1 . The homogeneous part of the proposed shallow flow system (4.1) is linearized with respect to the field variables. To avoid the explicit appearance of the algebraic relation (3.7) between $h_{p}$ and $h$, we consider the evolution equation for the bulk centre of mass $h$ directly. It obeys the same general conservation property as demonstrated in (3.26), but with a modified right-hand side. The hyperbolic properties, however, are invariant under this substitution. We get

$$
\boldsymbol{A} \partial_{t}\left(\begin{array}{c}
m \\
c \\
U \\
h
\end{array}\right)+\boldsymbol{B} \partial_{x}\left(\begin{array}{c}
m \\
c \\
U \\
h
\end{array}\right)=\left(\begin{array}{l}
0 \\
0 \\
0 \\
0
\end{array}\right)
$$

in which the matrices $\boldsymbol{A}$ and $\boldsymbol{B}$ are given by

$$
\boldsymbol{A} \equiv\left(\begin{array}{cccc}
1 & 0 & 0 & 0 \\
c & m & 0 & 0 \\
U & 0 & m & 0 \\
h & 0 & 0 & m
\end{array}\right), \quad \boldsymbol{B} \equiv\left(\begin{array}{cccc}
U & 0 & m & 0 \\
c U & m U & c m & 0 \\
U^{2}+g h & 0 & 2 m U & g m \\
0 & 0 & h & U
\end{array}\right) .
$$

The characteristic speeds $s_{i}$ and their eigenvectors $e_{i}(i \in\{1,2,3,4\})$ are

$$
\begin{gathered}
s_{1,2}=U \pm \sqrt{2 g_{y} h}, \quad s_{3,4}=U, \\
e_{1,2}=\left(\begin{array}{c}
c \\
c\left(U \pm \sqrt{2 g_{y} h}\right) \\
h
\end{array}\right), \quad e_{3}=\left(\begin{array}{c}
-m \\
0 \\
-m U \\
h
\end{array}\right), \quad e_{4}=\left(\begin{array}{l}
0 \\
c \\
0 \\
0
\end{array}\right) .
\end{gathered}
$$

Within the physical domain of positive masses the corresponding vertical centre of mass will be positive and system (4.1) has real characteristic speeds. It therefore comprises a hyperbolic first-order system of partial differential equations. In the case of zero masses the eigenvectors are not independent and the system is linearly degenerate. This occurs in all shallow flow models. Note also that for a uniform 
Nomenclature

$\varrho_{i}$ Component $i$ intrinsic density

$\rho_{i}$ Component $i$ density

$\rho$ Bulk density

$\boldsymbol{u}_{i}$ Component $i$ velocity

$\boldsymbol{u}$ Mass-averaged velocity

$v$ Volume-averaged velocity

d Slip velocity

$\sigma_{i}$ Effective component stress

$\sigma$ Effective bulk stress

g Gravitational acceleration

$\xi$ Inclination angle

$\boldsymbol{I}_{i}$ Momentum exchange

$\tau$ Viscous drag relaxation time

$\phi$ Maximum packing fraction

$\alpha=\rho_{f} / \varrho_{f}$ solids volume fraction

$\delta=\varrho_{f} / \varrho_{p}$ density ratio

$p$ Bulk pressure

$p_{e}$ Pore pressure

$p_{h}$ Hydrostatic fluid pressure
$H=H_{p}+H_{f}$
$H_{i}=m_{i} / \varrho_{i}$
$m_{i}=\int_{0}^{H} \rho_{i} \mathrm{~d} y$,
$m=\int_{0}^{H} \rho \mathrm{d} y$,
$c=m_{p} / m$
Surface height
Component $i$ equivalent height
Component $i$ depth-averaged mass
Bulk depth-averaged mass
$U=1 / m \int_{0}^{H} u_{x} \rho \mathrm{d} y$,
Particle mass fraction
$h=1 / m \int_{0}^{H} y \rho \mathrm{d} y$,
Depth-averaged horizontal velocity
$h_{i}=1 / m_{i} \int_{0}^{H} y \rho_{i} \mathrm{~d} y$,
Bulk centre of mass
Component $i$ centre of mass
$h^{(s)}$
Bulk equilibrium
centre of mass
$h_{i}^{(s)}$
$\mu_{1}$
D
$h_{p}^{\prime}=\frac{h_{p}}{H}-\frac{1}{2}$
Component $i$ equilibrium centre of mass
First Fourier decay time rate
Sedimentation velocity
Phase diffusion coefficient
Solids centre of mass
deviations
Characteristic shear
$\gamma$
$P e=V H / D$
Shallowness parameter
Péclet number
$p_{i}$

TABLE 1. The subscript $i$ indicates the component, $p$ for particles and $f$ for fluid.

vertical solids profile, the centre of mass corresponds to half the surface height $(h=H / 2)$, and the first pair of wave speeds reduces to the characteristic speeds of the shallow water system $s_{1,2}=U \pm \sqrt{g_{y} H}$.

\subsection{Travelling wave solutions}

We now look at a steady surge in a frame moving with constant velocity $-V$. In this reference frame the equations can be written

$$
\begin{aligned}
\frac{\mathrm{d}}{\mathrm{d} x}(m U) & =0, \\
\frac{\mathrm{d}}{\mathrm{d} x}(c m U) & =0, \\
\frac{\mathrm{d}}{\mathrm{d} x}\left(m U^{2}+g_{y} h m\right) & =\left(g_{x}-\mu g_{y}\right) m, \\
\frac{\mathrm{d}}{\mathrm{d} x}\left(h_{p} U\right) & =-\mu_{1}\left(h_{p}-h_{p}^{(s)}\right),
\end{aligned}
$$


and $x$ increases towards the tail of the avalanche. We are only considering regions where $m>0$ so that if $U=0$ anywhere then (4.7a) implies $U=0$. This system is then singular and we have $h_{p}=h_{p}^{(s)}$ and only one differential equation

$$
\frac{\mathrm{d}}{\mathrm{d} x}\left(g_{y} h m\right)=\left(g_{x}-\mu g_{y}\right) m \text {. }
$$

This can be solved for $m$ if we specify $c$ or solved for $c$ if we specify $m$. For example, we choose the pure material densities of fluid and solid to be $\varrho_{f}=1$ and $\varrho_{p}=2$ so that $\delta=1 / 2$ and for simplicity $g_{y}=1$. We assume that the flow is well mixed, $h_{p}^{(s)}=H / 2$, and that the head is solids rich and exponentially decays towards the tail, $c=\mathrm{e}^{-x / L}$. To ensure a force balance far up the slope we take

$$
\mu=g_{x}+\eta c
$$

where $\eta$ is a dimensionless parameter that determines the increase in friction with solids concentration. If we define $r=\exp (-x / L) \in[0,1]$ then we get the first-order ordinary differential equation

$$
2(2-r) \frac{\mathrm{d} m}{\mathrm{~d} r}=m+4 \eta,
$$

with solution

$$
m=4 \eta\left[\frac{A}{\sqrt{2-\exp (-x / L)}}-1\right],
$$

where $A$ is a constant of integration. For $1<A<\sqrt{2}$ this solution corresponds to finite-sized flows, since the mass is positive for $x \in\left[0,-L \log \left(2-A^{2}\right)\right]$. For $A>\sqrt{2}$ the flows approach constant mass as $x \rightarrow \infty$. The basic pattern resembles the solidsrich front shown in figure 1, where the high gradient counterbalances the increased friction towards the front. At the front itself $x=0$ the solution terminates and requires jump conditions, which we do not discuss further. This type of solution is similar to existing debris flow models since there is no vertical redistribution.

Next we consider finite $Q$ so that $U$ is always non-zero and the $h_{p}$ equation is no longer trivial. In this case we can eliminate $U=Q / m$ and (4.7a) implies $c=$ const.. We keep the same assumptions for $h_{p}^{(s)}$ and $\delta$ but make an additional assumption that there is a momentum balance so that $g_{x}=\mu$. Equation (4.7c) can then be integrated. To give

$$
h_{p}=\frac{3 A^{2}}{2 m}-\frac{4 Q^{2}}{m^{2}}-\frac{9 m}{8},
$$

where $A$ is a constant related to the momentum flux. As $h_{p}$ must satisfy consistency criteria it must be greater than $m / 8$ and less than $5 \mathrm{~m} / 8$. It is therefore convenient to define $\tilde{h}_{p}=\left(8 h_{p}-m\right) /(4 m)$, which must therefore lie between 0 and 1 . We also rescale $s$ by $A /(3 Q \sqrt{2})$, define $m=A \tilde{m}$ and let $Q=\sqrt{A^{3} \eta / 4}$. Using $(4.7 d)$ we then have

$$
\begin{aligned}
& \tilde{h}_{p}=\frac{1}{2}+\frac{3 \tilde{m}\left(1-\tilde{m}^{2}\right)-2 \eta}{\tilde{m}^{3}}, \\
& \frac{\mathrm{d} \tilde{m}}{\mathrm{~d} s}=-\tilde{m}^{2} \frac{3 \tilde{m}\left(1-\tilde{m}^{2}\right)-2 \eta}{\eta-\tilde{m}} .
\end{aligned}
$$

The behaviour of this system is governed by the roots of the right-hand side of (4.14). For $\eta>1 / 3$ there is one negative real root so any positive solution will evolve towards 
the pole at $\tilde{m}=\eta$ and cannot be continued. If a suitable jump condition exists such a solution might be physically reasonable. If $\eta<\sqrt{3}$ there are two positive real roots: one greater than $\eta$ and one, $m_{1}$, less than $\eta$. There is then a smooth solution where $m$ decreases towards zero as $s \rightarrow-\infty$ and increases towards $m_{1}$ as $s \rightarrow \infty$. We can obviously see from (4.13) that at this root, and indeed whenever $\tilde{m}$ is constant, we have $\tilde{h}_{p}=1 / 2$, indicating that as we move downslope the particle centre of mass evolves toward its equilibrium value. Moving back up the slope, however, $\tilde{h}_{p}$ decreases towards zero at $\tilde{m}^{3} \approx 4 \eta$ so the solution is invalid after this. In this solution we have balance between the momentum flux $m U^{2}$ and the centre of mass $h m$ subject to the centre of mass evolving according to sedimentation and resuspension. Such solutions can only occur in a model that explicitly allows for this.

\subsection{Homogeneous solutions}

Now we consider solutions where the $z$-dependence can be neglected, that is a zerothorder shallow water model. This is a reasonable approximation for the central section of many flows. The mass and concentration equations then imply that $m$ and $c$ are constant. We consider the case of a large amount of solid material $c=2 / 3$ overlain by water. Again we take $\varrho_{f}=1, \varrho_{p}=2, \phi=1, g_{y}=1$ and $H=1$. This mass fraction corresponds to a volume fraction of $1 / 2$ and $H=2 m / 3$. The equations of motion then reduce to two ordinary differential equations for $u$ and $h_{p}$ :

$$
\begin{gathered}
\frac{\mathrm{d} u}{\mathrm{~d} t}=g_{x}-\mu g_{y}, \\
\frac{\mathrm{d} h_{p}}{\mathrm{~d} t}=-\mu_{1}\left(h_{p}-H / 2\right) .
\end{gathered}
$$

As discussed in $\S \S 2.5$ and 2.8 it is possible to directly reconstruct the concentration profile from $h_{p}$ and $c$. This can be done using Fourier modes but this can lead to non-physical values of $\alpha$ that are either negative or $>\phi$. Instead we perform a reconstruction in terms of the logistic function. This is always monotonic and correctly bounded as well as being the exact steady solution. We take

$$
\alpha(t, z)=\frac{1}{1+\exp [A(t)((z / h)-(1 / 2))]},
$$

which gives the correct solids volume fraction of $1 / 2$. From (2.29) we have a relation between $h_{p}(t)$ and $A(t)$ :

$$
h_{p}=\frac{1}{2}+\frac{2}{A^{2}}\left[\operatorname{dil} \log \left(1+\mathrm{e}^{-A / 2}\right) \operatorname{dil} \log \left(1+\mathrm{e}^{A / 2}\right)\right]-\frac{2}{A} \log \left(\mathrm{e}^{A / 2}+\mathrm{e}^{-A / 2}\right) .
$$

The solids fraction at the base, $\alpha_{0}$, can then be determined by solving (4.18) for $A$ and substituting into (4.17). This dependence is shown in figure 5 as well as a simple approximation $\alpha_{0}=h_{p} / H\left(4 h_{p} / H-3\right)^{2}$ that matches the value and gradient at the end points. The work of Boyer, Guazzelli \& Pouliquen (2011) suggests that for solids fractions less than about half-maximum the particles support only a small fraction of the stress, but that this increases rapidly as maximum packing density is approached. Thus we write

$$
X= \begin{cases}0, & \alpha<1 / 2, \\ 2 \alpha-1, & \alpha>1 / 2,\end{cases}
$$




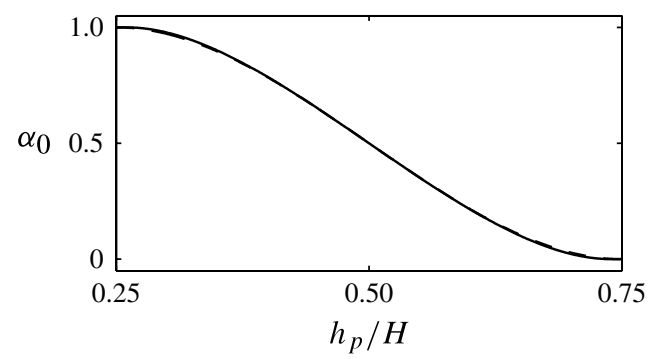

FIGURE 5. The relationship between the solids fraction at the basal surface, $\alpha_{0}$, and the relative centre of mass $h_{p} / H$ with a total solids volume fraction of $1 / 2$. The dashed line, which is almost indistinguishable, shows the approximation $\alpha_{0}=h_{p} / H\left(4 h_{p} / H-3\right)^{2}$.

so that the solids are fully suspended for $\alpha<1 / 2$ and that the pressure fraction increases until they take all the pressure when $\alpha=1$. We are then left to choose three more closures for $h_{p}^{(s)}, \mu$, and $\mu_{1}$. We assume that the rate of resuspension and sedimentation is constant so that $\mu_{1}=(1 / 4) \sqrt{g_{y} / H}$. For $\mu$ an accurate model might use the results from Boyer et al. (2011), but since our example is meant to merely be illustrative rather than quantitatively accurate we use a simpler approach. We suppose that there is a frictional component to the drag proportional to the particle pressure $\mu_{p} p_{p}=\mu_{p} X m g_{y}$ and we take $\mu_{p}=0.29$. We also assume a turbulent $\operatorname{drag} c_{D} u^{2} m / H$, where $c_{D}=0.3$ is a turbulent drag coefficient. The basal friction is then

$$
\mu=X \mu_{p}+c_{D} F r^{2},
$$

where $F r=u / \sqrt{H g_{y}}$. We define the steady velocity $u^{(s)}$ as the positive solution of $g_{y} \mu=g_{x}$ or zero. Finally at low speeds the particles must all sediment out, $h_{p}^{(s)}=H / 4$, whereas at high speeds the system will be well-mixed, $h_{p}^{(s)}=H / 2$. Therefore we take

$$
h_{p}^{(s)}=H / 2-(H / 4) \exp (-5 F r) .
$$

First we consider the initiation of a debris flow on a slope just steeper than the static angle of friction, $g_{x}=0.3$, that is initially at rest $u(0)=0$ and fully sedimented $h_{p}(0)=H / 4$. Figure 6 shows the results. Initially the flow slowly accelerates. The solids fraction at the base is close to 1 and basal friction is dominated by granular friction since $X$ is close to 1 . As the velocity increases the solids equilibrium centre of mass $h_{p}^{(s)}$ increases according to (4.21), and $h_{p}$ also increases with a small lag. As the solids fraction at the base decreases at around $t=5$ the equilibrium velocity $u^{(s)}$ rapidly increases and the flow accelerates until it reaches its steady state. The system is now well-mixed and $h=h_{p}^{(s)}=H / 2$ and $X=0$. The effective friction $\mu$ is still close to $g_{x}$ because the flow is at $u^{(s)}$, but it has switched from being frictional to turbulent drag.

From this state, even if the slope gets much shallower than the static friction angle, $g_{x}<\mu_{p} g_{y}$, it will continue to flow and maintain the particles in suspension down to around $g_{x}=0.12$. The results if the angle is reduced to $g_{x}=0.10$ are shown in figure 7. After an initial rapid relaxation of the velocity it only decays very slowly and is slaved to $u^{(s)}$, which is set by the solids fraction at the base. At around $t=60$ solid friction starts to become important and $u^{(s)}$ rapidly drops as the the fluid supports less of the weight of the particles. The flow then rapidly decelerates, coming to a complete 

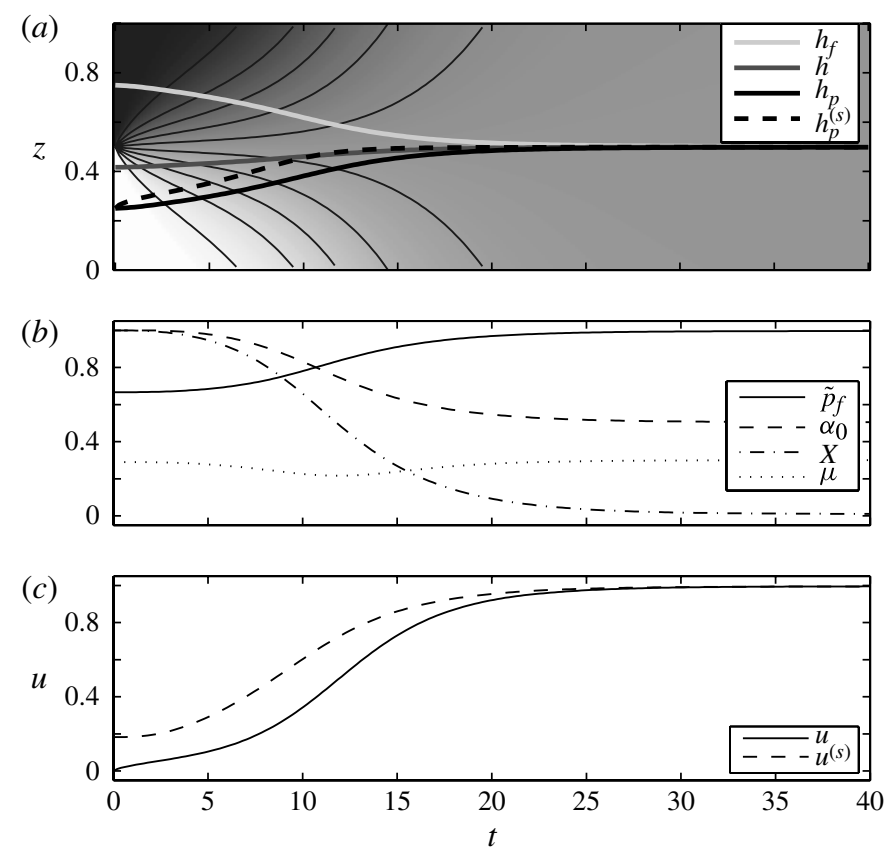

FIGURE 6. Numerical solution of the initiation of a debris flow with $g_{x}=0.3$. (a) Greyscale intensity shows particle volume fraction as a function of height and time. The lines show $h_{f}$, $h, h_{p}$ and $h_{p}^{(s)}$ as functions of time. (b) Quantities on the basal surface: pore pressure $p_{f}$, basal solids fraction $\alpha_{0}$, particle pressure fraction $X$ and total basal friction $\mu$. (c) Instantaneous velocity $u$ and steady velocity $u^{(s)}$.

stop at around $t=78$. The particles then continue to sediment until $h_{p}=H / 4$ and no further changes occur.

This shows that the model can describe the underlying physics of the resuspension and sedimentation processes and capture realistic physical effects such as periods of approximate equilibrium punctuated by rapid acceleration and deceleration driven by a switch in the dominant drag mechanism. These simple closures, used only for illustrative purposes, and the lack of $x$-dependence mean that quantitative comparison with the field data (figure 4) is not appropriate. Qualitative agreement can be seen, however, in the deviation of the fluid pore pressure from the normal stress. Figure 8 shows the reduction in the effective basal friction as the pore pressure rises.

\section{Discussion and conclusion}

Starting from general mixture theory we proposed an extended shallow fluid-solid flow model, that consists of evolution equations for depth-averaged mass, depthaveraged particle concentration, depth-averaged bulk velocity and the vertical centre of mass of the particles. We have discussed in detail the evolution equation for the particle centre of mass $h_{p}$ and shown how it adds additional terms to the other conservation equations. The centre of mass of the complete system is easily calculated from $h_{p}$ and we show that this is the correct length scale for defining the Froude number as it is this height that sets the wave speeds, not the surface height. The additional consideration of $h_{p}$ as a system variable is the real innovation of the 


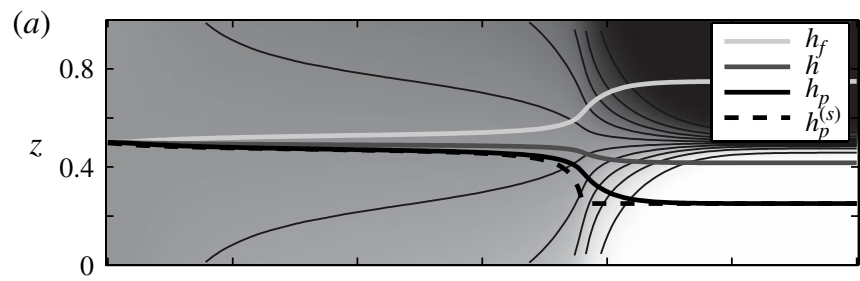

(b)
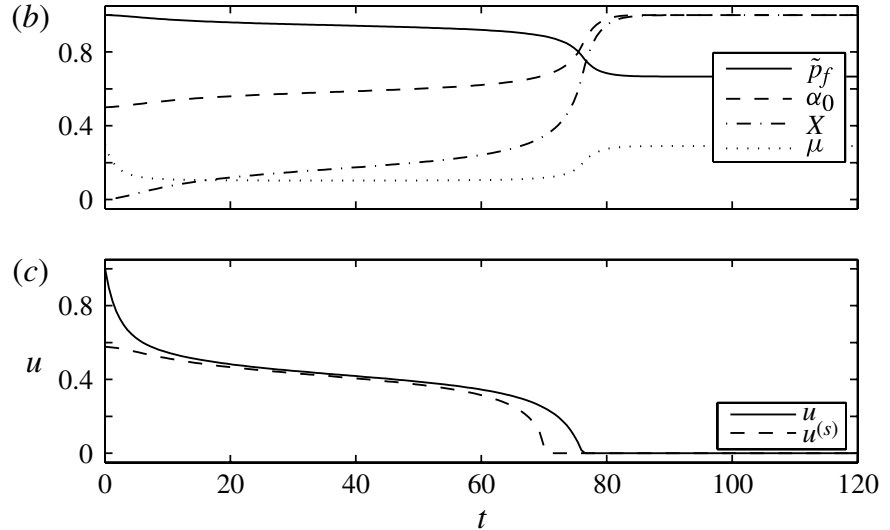

FIGURE 7. Numerical solution of the arrest of a debris flow with $g_{x}=0.10$. (a) Greyscale intensity shows particle volume fraction as a function of height and time. The lines show $h_{f}$, $h, h_{p}$ and $h_{p}^{(s)}$ as functions of time. (b) Quantities on the basal surface: pore pressure $p_{f}$, basal solids fraction $\alpha_{0}$, particle pressure fraction $X$ and total basal friction $\mu$. (c) Instantaneous velocity $u$ and steady velocity $u^{(s)}$.

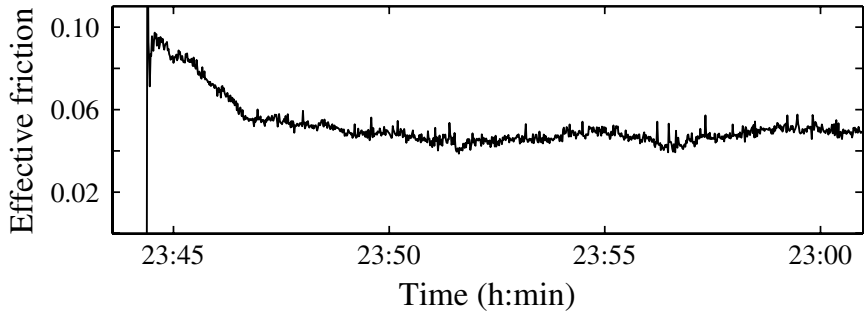

FIGURE 8. The plot shows the ratio of measured shear force over normal stress and can be interpreted as an apparent friction coefficient of the debris flow surge. In the front the friction is non-constant indicating that there basal drag is not fully described by dry Coulomb friction. Behind the flow head, the ratio of shear and normal force stays more or less constant.

proposed system; $h_{p}$ can be used to track the shifting balance between (re)suspension and sedimentation in fluid-solid mixtures within the simplified framework of depthaveraging. This clearly generalizes established geophysical shallow flow theories and offers a simple way of identifying different mixture regimes, e.g. a layered flow situation in which all particles are settled to the ground or a uniformly mixed one in which all particles are suspended in the fluid.

The resulting system is well-posed and constitutes a direct generalization of onephase shallow flow theory. We have briefly demonstrated how resuspension and 
sedimentation are included in the model. We have restricted ourselves to a very simple setting in two spatial dimensions, assumed a flat topography and neglected any entrainment processes at the basal surface. Likewise we avoided any discussion of the controversial question of the correct basal friction law. Considering extended shallow flow models in realistic topography is therefore a planned next step.

In debris flows an important observable in both real-scale and laboratory experiments is the basal fluid pore pressure. Most shallow multi-component flow approximations for debris flows face the limitation of a fixed vertical mixture density, which we overcome in our approach. We show how the basal pore pressure directly depends on $h_{p}$ and how this can be positive or negative depending on whether particles are sedimenting or being resuspended.

The proposed extension to the shallow flow theory can be embedded in a even more general theory by taking additional moments of the fundamental balance laws. A possible application of this would be an analysis of the effect of entrainment on velocity profiles for example. The concept is also very general and not at all restricted to the case of a binary fluid-solid mixture. A natural next step therefore is an extension to more constituents, for example polydisperse granular flows with or without a liquid.

\section{Acknowledgements}

The authors would like to thank the Swiss Federal Office of the Environment (BAFU) and the Swiss National Science Foundation (SNF) for their financial support. J.N.M. was supported by an ARF from the UK Engineering and Physical Sciences Research Council.

\section{REFERENCES}

BArtelt, P., SAlm, B. \& Gruber, U. 1999 Calculating dense-snow avalanche runout using a voellmy-fluid model with active/passive longitudinal straining. J. Glaciol. 45 (150), 242-254.

BenNy, D. J. 1973 Some properties of long nonlinear waves. Stud. Appl. Maths 52, 45-50.

BERRES, S., BÜRGER, R. \& TORY, E. M. 2005 On mathematical models and numerical simulation of the fluidization of polydisperse suspensions. Appl. Math. Model. 29, 159-193.

Berzi, D. \& Jenkins, J. T. 2009 Steady inclined flows of granular-fluid mixtures. J. Fluid Mech. 641, 359-387.

Boyer, F., Guazzelli, É. \& Pouliquen, O. 2011 Unifying suspension and granular rheology. Phys. Rev. Lett. 107, 188301.

Cassar, C., Nicolas, M. \& Pouliquen, O. 2005 Submarine granular flows down inclined planes. Phys. Fluids 17, 103301.

Christen, M., Kowalski, J. \& BARTelt, P. 2010 Ramms: numerical simulation of dense snow avalanches in three-dimensional terrain. Cold Reg. Sci. Technol. 63 (1-2), 1-14.

DENLINGER, R. P. \& IVERSON, R. M. 2001 Flow of variably fluidized granular masses across three-dimensional terrain 2. Numerical predictions and experimental tests. J. Geophys. Res. 106 (B1), 553-566.

Drew, D. A. \& Passman, S. L. 1998 Theory of Multicomponent Fluids. Springer.

GRAY, J. M. N. T. 2002 Rapid granular avalanches. In Dynamic Response of Granular and Porous Material Under Large and Catastrophic Deformations, Lecture Notes in Applied and Computational Mechanics, vol. 11. pp. 3-42. Springer.

Gray, J. M. N. T. \& Chugunov, V. A. 2006 Particle-size segregation and diffusive remixing in shallow granular avalanches. J. Fluid Mech. 569, 365-398.

Gray, J. M. N. T. \& KoKelaAR, B. P. 2010 Large particle segregation, transport and accumulation in granular free-surface flows. J. Fluid Mech. 652, 105-137. 
Gray, J. M. N. T. \& Thornton, A. R. 2005 A theory for particle size segregation in shallow granular free-surface flows. Proc. R. Soc. Lond. A 461, 1447-1473.

IshiI, M. \& HIBIKI, T. 2006 Thermo-fluid Dynamics of Two-phase Flow. Springer.

IVERson, R. M. 1997 The physics of debris flows. Rev. Geophys. 35 (3), 245-296.

IVERSON, R. M. 2009 Elements of an improved model of debris-flow motion. In Invited Contribution to Powders and Grains 2009 Conference. American Physical Society.

IVERson, R. M. \& DENLINGER, R. P. 2001 Mechanics of debris flows and debris-laden flash floods. In Seventh Federal Interagency Sedimentation Conference, pp. IV-1-IV-8.

IVERson, R. M. \& MAJOR, J. J. 1999 Debris-flow deposition: effects of pore-fluid pressure and friction concentrated at flow margins. Geol. Soc. Am. Bull. 111 (10), 1424-1434.

LAVOREL, G. \& LE BARS, M. 2009 Sedimentation of particles in a vigorously convecting fluid. Phys. Rev. E 80 (4), 046324.

McArdell, B., BARTelt, P. \& Kowalski, J. 2007 Field observations of basal forces and fluid pore pressure in a debris flow. Geophys. Res. Lett. 34, L07406.

McArdell, B. W., Zanuttigh, B., Lamberti, A. \& Rickenmann, D. 2003 Systematic comparison of debris flows at the Illgraben torrent, Switzerland. In Debris-Flow Hazards Mitigation: Mechanics, Prediction and Assessment, pp. 647-657. Millpress.

Pailha, M. \& Pouliquen, O. 2008 Initiation of underwater granular avalanches: influence of the initial volume fraction. Phys. Fluids 20, 111701.

Pelanti, M., Bouchut, F. \& Mangeney, A. 2008 A roe-type scheme for two-phase shallow granular flows over variable topography. ESAIM: Math. Model. Num. Anal. 42, 851-885.

Pitman, E. B. \& LE, L. 2005 A two-fluid model for avalanche and debris flow. Phil. Trans. R. Soc. Lond. 363, 1573-1601.

Pudasaini, S. P. \& Hutter, K. 2007 Avalanche Dynamics-Dynamics of Rapid Flows of Dense Granular Avalanches. Springer.

Richardson, J. F. \& ZAKI, W. N. 1954 Sedimentation and fluidisation. Part 1. Trans. Inst. Chem. Engng 32, 35-53.

SAVAge, S. B. \& HutTer, K. 1989 The motion of a finite mass of a granular material down a rough incline. J. Fluid Mech. 199, 177-215.

SAvage, S. B. \& Hutter, K. 1991 The dynamics of avalanches of granular materials from initiation to runout. part 1: Analysis. Acta Mechanica 86, 201-223.

Scheid, B., Ruyer-Quil, C. \& Manneville, P. 2006 Wave patterns in film flows: modelling and three-dimensional waves. J. Fluid Mech. 562, 183-222.

Thornton, A. R., Gray, J. M. N. T. \& HogG, A. J. 2006 A three-phase mixture theory for particle size segregation in shallow granular free-surface flows. J. Fluid Mech. 550, 1-25.

Wieland, M., Gray, J. M. N. T. \& HutTer, K. 1999 Channelized free-surface flow of cohesionless granular avalanches in a chute with shallow lateral curvature. J. Fluid Mech. 392, 73-100. 